\title{
Enzalutamide, an oral androgen receptor inhibitor for treatment of castration-resistant prostate cancer
}

\author{
Cora N Sternberg*,1 \\ ${ }^{1}$ Division of Medical Oncology, Weill Cornell Medical Center, New York, NY 10021, USA \\ *Author for correspondence: Tel.: +1 646962 2072; Fax: +1 646962 1603; cns9006@med.cornell.com
}

Androgen receptor (AR) signaling is a key pathway in prostate cancer, and patients are initially treated with androgen deprivation therapy. Patients who have stopped responding to androgen deprivation therapy are considered to have castration-resistant prostate cancer (CRPC), which is still dependent on AR signaling. Enzalutamide, an orally available AR inhibitor, was initially approved by the US FDA for the treatment of patients with metastatic CRPC who have previously received docetaxel. The indication was subsequently extended to include all patients with metastatic CRPC, and most recently to include patients with nonmetastatic CRPC. This review summarizes the body of evidence supporting enzalutamide efficacy and safety in CRPC.

First draft submitted: 18 December 2018; Accepted for publication: 30 January 2019; Published online: 8 March 2019

Keywords: androgen receptor $\bullet$ castration-resistant prostatic cancer $\bullet$ enzalutamide $\bullet$ patient-reported outcomes $\bullet$ prostate-specific antigen $\bullet$ safety $\bullet$ treatment outcome

Advanced prostate cancer (PC) progresses along a continuum, culminating in the lethal phenotype of castration-resistant PC (CRPC), defined as disease reactivation despite a serum testosterone level of $<50 \mathrm{ng} / \mathrm{dl}$ $(<1.7 \mathrm{nmol} / \mathrm{l})$ [1]. As is true for earlier disease stages, tumor growth and progression in CRPC is often dependent on androgen receptor (AR) signaling, which is associated with increased production and circulatory levels of prostate-specific antigen (PSA) in the majority of, but not all, patients [2].

AR overexpression is one of the many mechanisms of resistance to androgen-deprivation therapy (ADT), with elevated gene copy number reported in up to $80 \%$ of tumors [3-6]. In a xenograft model of PC, AR overexpression amplified signal output from low levels of residual ligand and led to a paradoxical 'antagonist-agonist' conversion in the presence of first-generation antiandrogens [5]. Further research confirmed that the partial agonist properties of first-generation antiandrogen agents manifest when PC cells overexpress the AR. This has been evidenced clinically by the antiandrogen withdrawal response whereby serum PSA levels decline upon discontinuation of antiandrogens $[7,8]$. Increased levels of intratumoral androgens are also detected among patients with progressive PC [9]. Taken together, these observations provided a rationale for developing new agents capable of more efficient inhibition of AR signaling in CRPC $[10,11]$.

Enzalutamide (Xtandi ${ }^{\circledR}$ [formerly MDV3100]; Pfizer Inc., NY, USA, and Astellas Pharma, Inc., IL, USA) is an orally available, nonsteroidal, second-generation antiandrogen that acts on multiple steps in the AR signaling pathway [12]. Here, we provide an overview of the development of enzalutamide for PC within the context of an evolving understanding of the mechanisms underlying AR signaling and disease progression.

\section{Epidemiology \& current therapeutic options}

PC accounted for more than 350,000 deaths worldwide in 2018, representing the fifth leading cause of male cancer-related death $(\sim 6.6 \%$ of total) [13]. Metastases are present in approximately $5 \%$ of patients at the time of PC diagnosis [14].

Initial hormonal treatment strategies that deplete testosterone and inhibit AR signaling are effective in most patients with advanced PC, but almost all will eventually progress to CRPC [15]; 10-20\% progress within 5 years of follow-up [16]. Approximately one-quarter of men diagnosed with PC are $\geq 75$ years of age, yet they are overrepresented with respect to metastases at diagnosis (48\%) and PC-specific deaths (53\%) [17].

Future Medicine 
Table 1. Approved noncytotoxic therapies for castration-resistant prostate cancer in the European Union and USA.

\begin{tabular}{|c|c|c|c|c|c|}
\hline Therapy (trade name) & Administration route & $\begin{array}{l}\text { Dosing } \\
\text { frequency }\end{array}$ & Dose level & Approval year & Indication(s) \\
\hline Enzalutamide (Xtandi) & Oral & Daily & $160 \mathrm{mg}$ & $\begin{array}{l}2012 \text { (US) } \\
2013 \text { (EU) }\end{array}$ & $\mathrm{M} 0$ and $\mathrm{M} 1 \mathrm{CRPC}$ \\
\hline Abiraterone acetate (Zytiga) & Oral $^{\dagger}$ & Daily & $1000 \mathrm{mg}$ & 2011 & $\mathrm{mCRPC}$ and $\mathrm{mHSPC}$ \\
\hline Sipuleucel-T (Provenge) & Intravenous infusion & Biweekly & $\begin{array}{l}\text { Three complete } \\
\text { doses } \ddagger\end{array}$ & $\begin{array}{l}2010 \text { (US) } \\
2013 \text { (EU) } \\
\text { Withdrawn } 2015 \text { (EU) }\end{array}$ & $\begin{array}{l}\text { Asymptomatic or minimally } \\
\text { symptomatic mCRPC }\end{array}$ \\
\hline Radium-223 dichloride (Xofigo) & Intravenous injections & 4 weekly & 6 injections $^{\S}$ & 2013 & $\begin{array}{l}\text { CRPC, symptomatic bone metastases } \\
\text { and no known visceral metastatic } \\
\text { disease }\end{array}$ \\
\hline Apalutamide (Erleada) & Oral & Daily & $240 \mathrm{mg}$ & 2018 (US) & MO CRPC \\
\hline \multicolumn{6}{|c|}{$\begin{array}{l}\dagger \text { Coadministered with prednisone } 5 \mathrm{mg} \text { orally twice daily. } \\
¥ \text { Each dose contains } 50 \text { million autologous CD54+ cells activated with prostatic acid phosphatase (PAP)-granulocyte-macrophage colony-stimulating factor (GM-CSF). } \\
\S 50 \mathrm{kBq}(1.35 \text { microcurie) per kg bodyweight. } \\
\text { CRPC: Castration-resistant prostate cancer; M0: Nonmetastatic; M1: Metastatic; mCRPC: Metastatic castration-resistant prostate cancer; mHSPC: Metastatic hormone-sensitive } \\
\text { prostate cancer; EU: European Union. }\end{array}$} \\
\hline
\end{tabular}

Before 2010, the median survival from the time of CRPC diagnosis was 19 months with docetaxel, and only symptomatic relief was available for commonly occurring skeletal-related events (SREs), such as bone pain, fractures, spinal cord compression and vertebral collapse [16,18]. Introduction of four new noncytotoxic therapies, an immunotherapy (sipuleucel-T), androgen-signaling inhibitors (abiraterone acetate [abiraterone] and enzalutamide) and a bone-targeting radiopharmaceutical (radium-223 dichloride), has extended overall survival (OS) in the metastatic setting by approximately $4-5$ months and provided varying levels of improvement in health-related quality of life (HRQoL) and pain mitigation (Table 1) [19]. Cabazitaxel chemotherapy prolongs OS in patients with metastatic CRPC (mCRPC) progressing after docetaxel.

More recent efforts to effect change earlier in high-risk patients with nonmetastatic castration-resistant prostate cancer (nmCRPC) have been fruitful, with enzalutamide and apalutamide producing a range of benefits, including significant prolongation of metastasis-free survival (MFS) [20,21]. The progression to CRPC is associated with a high risk of metastasis, and up to one-third of patients manifest metastatic disease within 2 years. MFS, a relatively new trial end point recognized by the US FDA as a clinically relevant end point in nmCRPC [22], has been defined as time from randomization to radiographic progression or death (any cause) and provides a disease-state specific measure of progression that avoids confounding by multiple subsequent therapies [23]. Of note, delaying the time to metastasis may prolong survival and forestall tumor-related complications [1]. As the first agent in its class developed to specifically target the AR, enzalutamide and its trial program have contributed meaningfully to understanding drivers of tumorigenesis.

Enzalutamide

Enzalutamide (4-(3-(4-cyano-3-(trifluoromethyl)phenyl)-5,5-dimethyl-4-oxo-2-thioxoimidazolidin-1-yl)-2fluoro-N-methylbenzamide) was synthesized in 2006 using the nonsteroidal agonist RU-59063 as a starting chemical scaffold [12]. This starting compound was selected on the basis of its relatively high affinity and selectivity for AR over other nuclear hormone receptors [5].

A structure-activity relationship study carried out on a series of thiohydantoins and their analogs, which included chemical modifications to improve serum half-life and oral bioavailability, resulted in the identification of a lead compound RD162 [12,24]. The RD162 has a half maximal inhibitory concentration nearly eight-times lower than that of bicalutamide, excellent activity in CRPC cells engineered to express higher levels of wild-type $\mathrm{AR}$ (LNCaP/AR), and a pharmacokinetic profile that facilitates oral administration [24]. Further modification of RD162 yielded enzalutamide (RD162'; MDV3100), which was chosen as the clinical candidate from this series because it had slightly greater activity in hormone-refractory LNCaP/AR cells and greater ease of manufacture (Figure 1) [24].

\section{Pharmacology}

Enzalutamide competitively inhibits androgen binding to the AR, nuclear translocation of the AR, DNA binding and coactivator recruitment (Figure 2) [12,24,25]. This impairment of AR DNA binding and AR transcription 


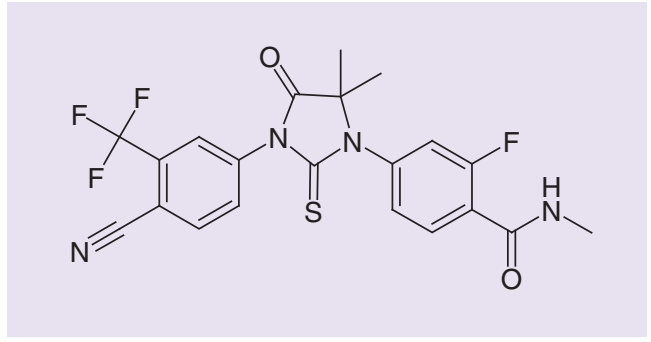

Figure 1. Chemical structure of enzalutamide. Molecular weight: 464.44 atomic mass units.

\begin{tabular}{|c|c|}
\hline Enzalutamide & $\mathrm{N}$-desmethyl enzalutamide \\
\hline 16.6 & 12.7 \\
\hline 11.4 & 13.0 \\
\hline $1(0.5-3.0)$ & $4(0-24)$ \\
\hline 300 & 193 \\
\hline $97-98$ & 95 \\
\hline $6.0(2.8-10.2)$ & $8.6(\% \mathrm{CV}, 21 \%)^{\S}$ \\
\hline $0.56(0.33-1.02)$ & NR \\
\hline $71 \%$ urine; $14 \%$ & \\
\hline \multicolumn{2}{|c|}{$\begin{array}{l}\text { †150 mg dose. } \\
\text { ¥After single-dose administration. } \\
\text { \$In healthy volunteers. } \\
\text { IOnly trace levels of unchanged enzalutamide and N-desmethyl enzalutamide were recovered. } \\
\text { AUC: Area under the plasma concentration-time curve during the 24-h dosing interval calculated by the linear trapezoidal rule; CV: Coefficient of variation; CL: Plasma clearance; Cmax: } \\
\text { Maximum plasma concentration; Cmin: Minimum plasma concentration; F: Bioavailability; mCRPC: Metastatic castration-resistant prostate cancer; NR: Not reported; SS: Steady state; } \\
\text { t1/2: Terminal elimination half-life; Tmax: Time to Cmax; Vd: Volume of distribution. } \\
\text { Data previously presented [29,30]. }\end{array}$} \\
\hline
\end{tabular}

complex assembly appears to be the basis for the absence of agonistic effects with enzalutamide. Partial agonism with first-generation antiandrogens, such as bicalutamide, has been attributed to aberrant recruitment of coactivators to transcription complexes and consequent gene activation. Further, it is hypothesized that the lack of AR agonism with enzalutamide is related to specific conformational changes induced upon receptor binding that differ from those with bicalutamide [12].

Enzalutamide has a half-life of 5.8 days in patients with mCRPC (Table 2) that allows for once-daily oral administration. The molecule attains a steady state by day 28 , accumulates 8.3 -fold with once-daily dosing, exhibits dose proportionality from 30 to $360 \mathrm{mg}$ /day and has low intersubject variability $(\leq 30 \%)[27,28]$. Food does not have a meaningful effect on total systemic exposure to enzalutamide or its active metabolite, $\mathrm{N}$-desmethyl enzalutamide [27]. Enzalutamide is eliminated primarily by hepatic metabolism, with renal excretion an insignificant elimination pathway for both enzalutamide and $\mathrm{N}$-desmethyl enzalutamide [27]. No enzalutamide dosage adjustment is required for patients with mild, moderate or severe hepatic impairment [27,29].

Drug-drug interactions with enzalutamide may require careful evaluation of concomitant medications and dose adjustments. Strong cytochrome P450 (CYP) 2C8 inhibitors can increase composite systemic exposure of enzalutamide plus $\mathrm{N}$-desmethyl enzalutamide by 2.2 -fold, and enzalutamide is a moderate CYP2C9 and CYP2C19 inducer and a strong CYP3A4 inducer [31]. Therefore, strong CYP2C8 inhibitors, strong CYP3A4 inducers, and CYP3A4, CYP2C9 and CYP2C19 substrates with a narrow therapeutic index should not be coadministered with enzalutamide [29]. If coadministration with CYP2C8 inhibitors cannot be avoided, the product label recommends dose reduction from 160 to $80 \mathrm{mg}$ once daily. Similarly, a dose increase from 160 to $240 \mathrm{mg}$ once daily is recommended when enzalutamide is taken with strong CYP3A4 inducers. Given the high pill burden in the majority of elderly patients treated with enzalutamide, clinicians should be aware of alternative treatment strategies for comorbidities. It may be noted that randomized clinical studies investigating efficacy with enzalutamide at reduced or increased doses have not been performed. However, based on the results of the Phase III trial in patients 


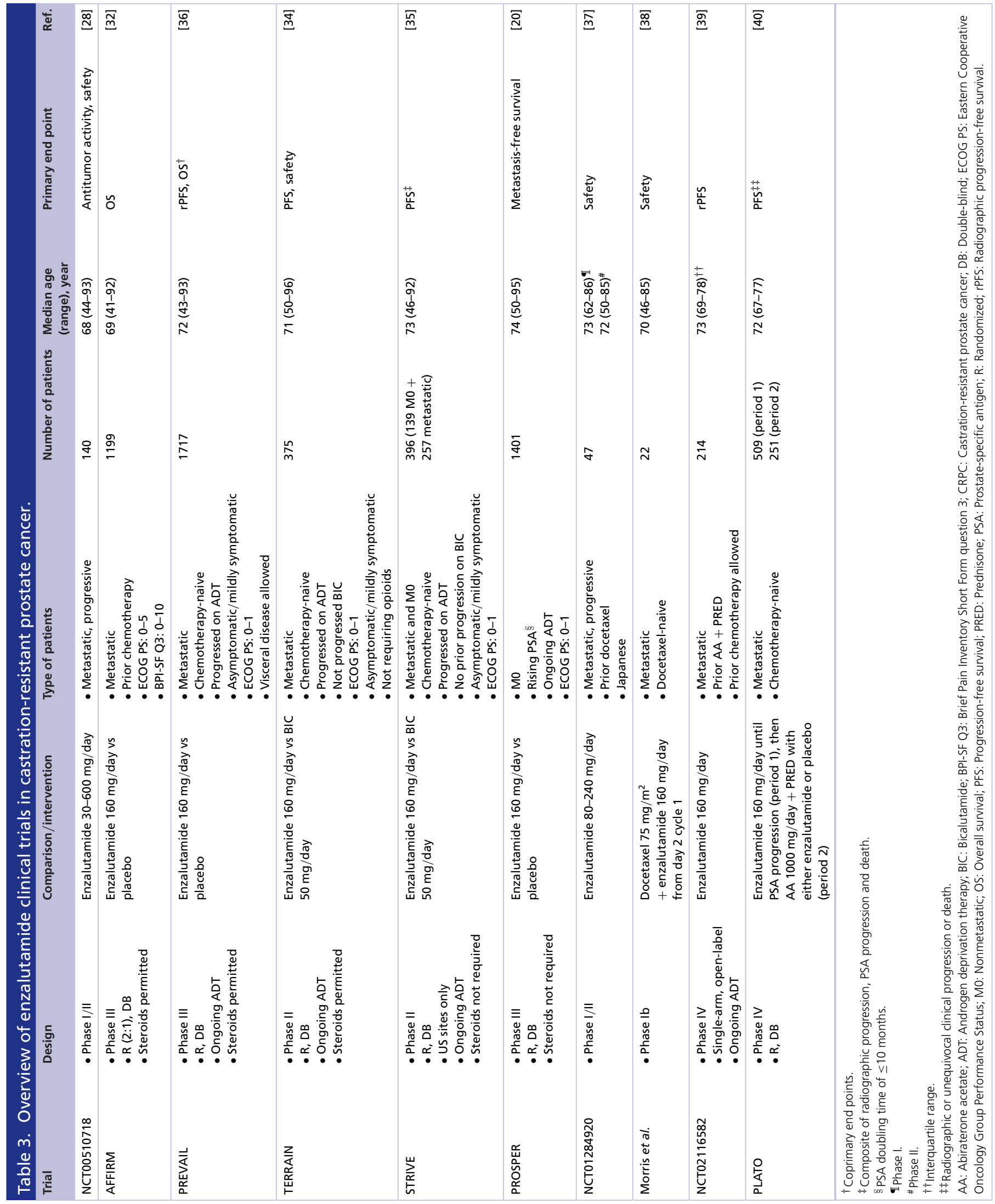


with mCRPC, there is no apparent exposure-response relationship for the efficacy end point of OS within a single fixed oral dose of $160 \mathrm{mg} /$ day [27].

\section{Clinical efficacy in patients with CRPC}

Clinical trials in PC vary in design as a function of disease state, presence of metastases, and prior therapies (Figure 3) and enzalutamide is being actively evaluated across the spectrum of disease biology. The double-blind, placebo-controlled, Phase III AFFIRM trial (NCT00974311) of patients with mCRPC, who had received previous docetaxel, [32] was initiated after enzalutamide showed encouraging clinical activity and acceptable tolerability among chemotherapy-experienced and chemotherapy-naive patients with progressive mCRPC in a dose-ranging Phase I/II trial [28].

The Phase III PREVAIL trial (NCT01212991) was then conducted based on the need for effective, less toxic treatments in the prechemotherapy setting given that not all mCRPC patients are eligible for or require docetaxel chemotherapy because of pre-existing medical conditions or concerns about toxicity [33].

Two Phase II trials were subsequently conducted to evaluate ADT in combination with enzalutamide versus bicalutamide in mCRPC (TERRAIN; NCT01288911) [34] and in both mCRPC and nmCRPC (STRIVE; NCT01664923) [35]. At the time TERRAIN and STRIVE were initiated, the active comparator bicalutamide was commonly used with ADT to treat CRPC despite concerns about short-lived efficacy and disease progression with partial AR agonism.

The double-blind, placebo-controlled, Phase III PROSPER trial (NCT02003924) was conducted to determine if enzalutamide could delay progression among nmCRPC patients with rapidly rising PSA levels, a population at high risk for metastasis [20]. Two ongoing Phase III trials with enzalutamide should provide valuable information on early AR inhibition in combination with standard treatments in the metastatic hormone-sensitive PC setting. ARCHES (NCT02677896) is investigating enzalutamide with ADT versus ADT alone in men with hormonesensitive disease and prior chemotherapy. ENZAMET (NCT02446405) will compare enzalutamide with ADT versus conventional antiandrogens (such as bicalutamide) plus ADT. A summary of enzalutamide's efficacy in CRPC is provided in (Table 3).

\section{Metastatic disease: Phase III trials of enzalutamide}

In AFFIRM and PREVAIL, enzalutamide produced significantly greater improvements in survival outcomes and responses than placebo and was associated with benefits across all secondary end points (Table 4) [32,36]. Both trials were stopped after planned interim analyses revealed a clear benefit in favor of enzalutamide over placebo for their respective primary end points [34,36].

\section{AFFIRM: postchemotherapy $m$ CRPC}

In AFFIRM, 1199 docetaxel-experienced patients across 156 sites in 15 countries were randomly assigned 2:1 to receive either enzalutamide $(n=800)$ or placebo $(n=399)$ [32]. Median treatment duration at the interim analysis was 8.3 months in the enzalutamide group and 3.0 months in the placebo group. At the time of the interim analysis, median OS (primary end point) was longer in the enzalutamide group than in the placebo group (18.4 vs 13.6 months, hazard ratio [HR]: 0.63; 95\% CI: 0.53-0.75; $\mathrm{p}<0.001)$. On this basis, AFFIRM was unblinded and eligible patients from the placebo group were switched to enzalutamide [32]. Enzalutamide demonstrated superior efficacy to placebo for secondary end point measures, including PSA level, soft-tissue response rates, times to PSA progression, radiographic progression-free survival ( $\mathrm{rPFS}$ ) and first SRE [32].

The OS benefit was generally consistent across all subgroups, including age, pain intensity, geographic region, type of disease progression at entry, number of bone metastases, PSA level and lactate dehydrogenase (LDH) level at baseline [32]. Results from AFFIRM helped to establish the critical contribution of androgen signaling to disease progression in PC despite low levels of circulating androgens and previous ADT.

In both patient age groups ( $\geq 75$ and $<75$ years) median OS was significantly better with enzalutamide than with placebo, as was median rPFS [41]. Enzalutamide was also associated with greater improvements in OS, rPFS, and time to PSA progression than placebo regardless of disease burden, as assessed by baseline PSA quartiles [46].

Acknowledging that the presence of visceral disease has prognostic significance, outcomes were analyzed in patients with liver versus lung disease in AFFIRM [47]. In 92 patients with liver metastases, enzalutamide was associated with a lower risk for radiographic progression (HR: 0.645; 95\% CI: 0.413-1.008), higher 12-month OS (37.7 vs $20.6 \%)$ and rPFS (11.6 vs $3.0 \%)$ rates and higher PSA response rates (35.1 vs $4.8 \%)$ than placebo [47]. 


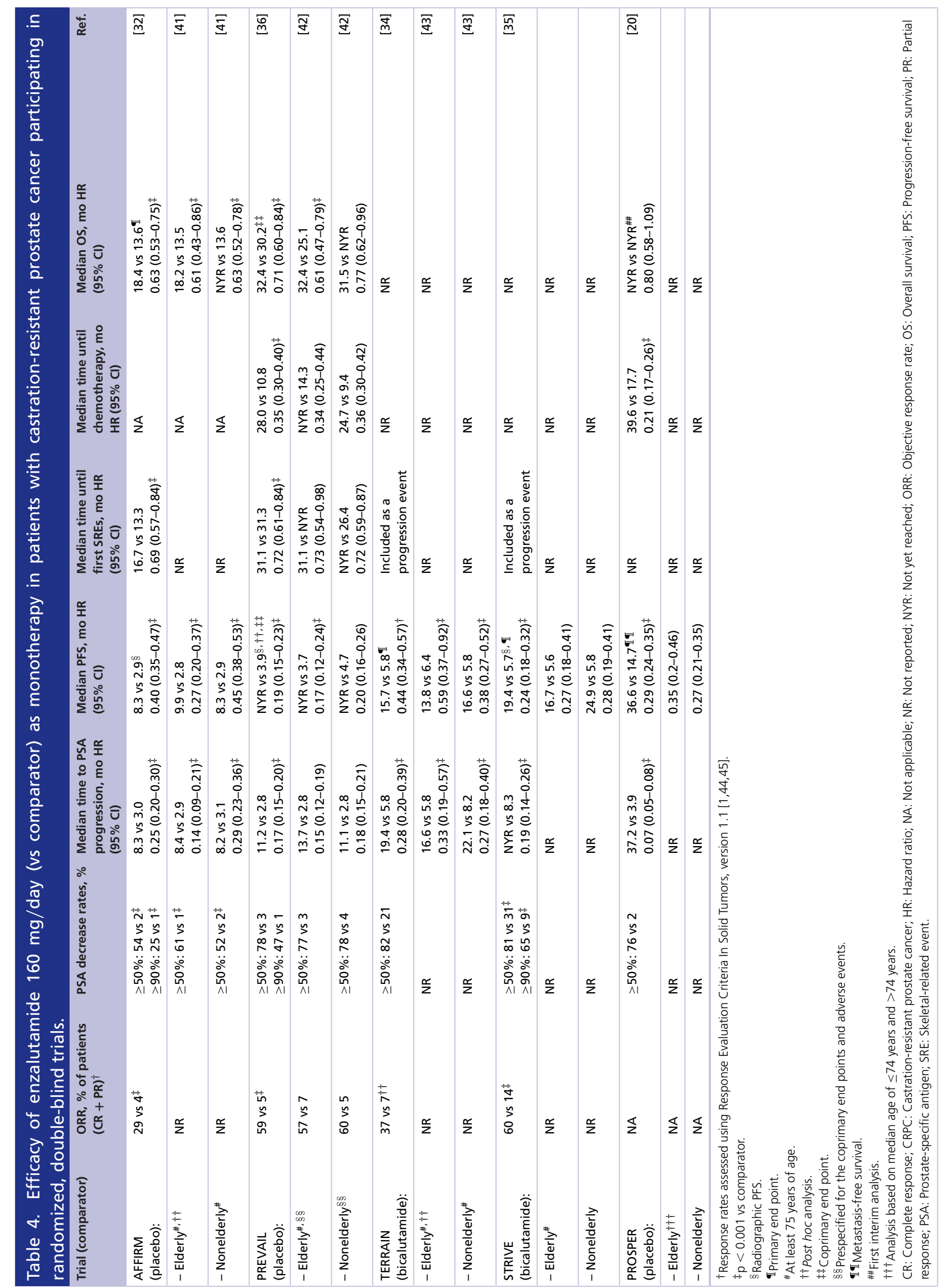



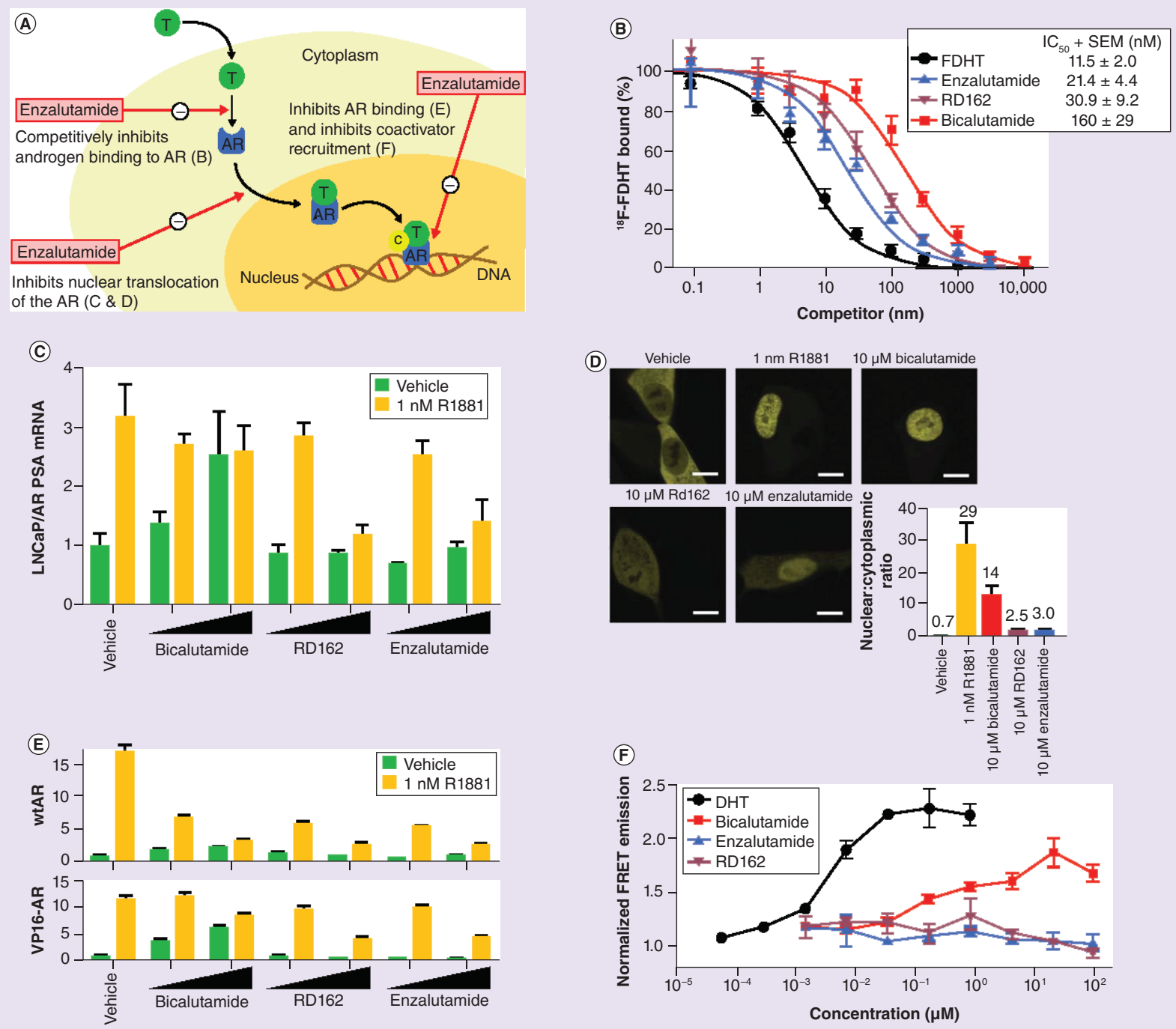

Figure 2. Enzalutamide's mechanism of action and key preclinical data. (A) Mechanism of action of enzalutamide in the AR signaling pathway [26]. (B) Enzalutamide binds AR in LNCaP/AR cells with five- to eightfold greater affinity than bicalutamide and only two- to threefold reduced affinity relative to the derivative of the native ligand $18 \mathrm{~F}-16$ - $\beta$-fluoro-5- $\alpha$-dihydrotestosterone [12]. (C) Real-time PCR quantification of chromatin immunoprecipitation analysis of AR in LNCaP cells shows that enzalutamide inhibits AR nuclear translocation at clinically relevant concentrations and is more effective than bicalutamide at inhibiting agonist-induced AR nuclear translocation [12]. (D) Confocal microscopy in live LNCaP cells shows that AR-enhanced yellow fluorescent protein was predominantly cytoplasmic in the absence of androgen, but largely nuclear after treatment with R1881 or bicalutamide. The ratio of nuclear versus cytoplasmic AR in RD162- or MDV3100-treated cells was approximately fivefold reduced relative to bicalutamide [12]. (E) The nuclear AR in RD162 or MDV3100-treated cells is not competent for DNA binding, as evidenced by a VP16-AR fusion protein-mediated activation of AR-dependent luciferase [12]. (F) DHT and bicalutamide both promoted dose-dependent interaction of the AR ligand-binding domain with an FxxLF motif-containing coactivator proteins, whereas RD162 and MDV3100 did not [12].

AR: Androgen receptor; DHT: Dihydrotestosterone; FRET: Fluorescence resonance energy transfer; IC 50 : Half maximal inhibitory coefficient; LNCaP/AR: CRPC cells engineered to express higher levels of wild-type AR (wtAR); SEM: Standard error of the mean; T: Testosterone. Panel A adapted with permission from [26] (c) Dove Medical Press Limited, under a Creative Commons Attribution - NonCommercial (unported, v3.0) license (2015).

Panels B-F adapted with permission from [12] ๔ AAAS (2009).

In the 104 patients with lung metastases, enzalutamide was associated with a lower risk of radiographic progression (HR: 0.386; 95\% CI: 0.259-0.577), higher 12 -month OS (65.1 vs 55.3\%) and rPFS (30.9 vs 8.2\%) rates, greater time to PSA progression (HR: 0.358; 95\% CI: 0.204-0.627) and better PSA response rates (52.1 vs 4.9\%) than placebo, and the risk of death was lowered (HR: 0.848; 95\% CI: 0.510-1.410) [47]. The survival benefits seen in AFFIRM across all patient subgroups confirms the efficacy of AR inhibition in this heterogeneous population. 


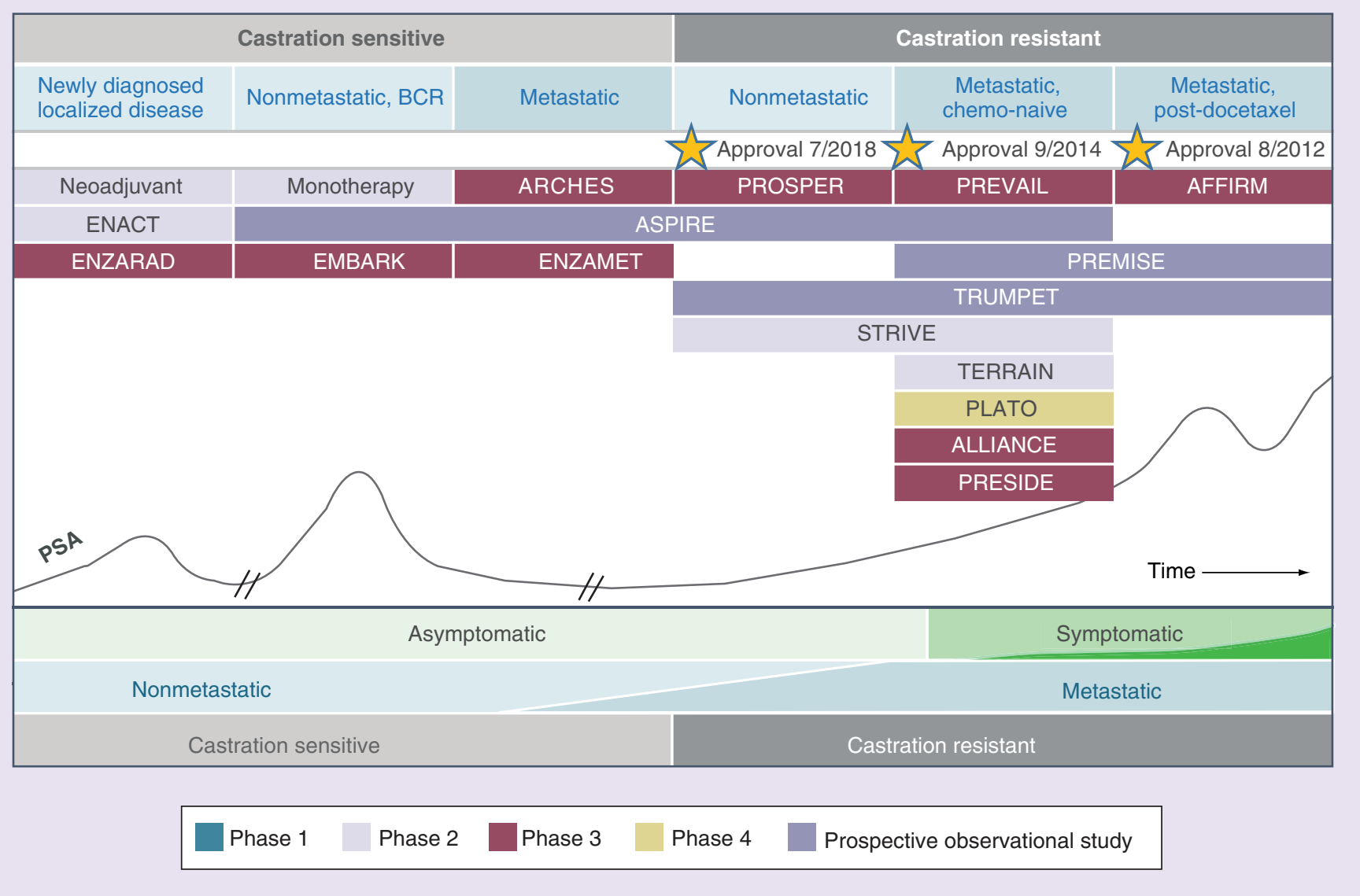

Figure 3. Key enzalutamide clinical trials along the spectrum of prostate cancer disease biology. BCR: Biochemical recurrence; PSA: Prostate-specific antigen.

\section{PREVAIL: chemotherapy-naive $m C R P C$}

PREVAIL was designed to evaluate enzalutamide earlier in patients who had not yet received chemotherapy and were asymptomatic or mildly symptomatic. PREVAIL introduced the coprimary end points of OS and rPFS. In contrast to a final OS end point, rPFS provided a measure linked to interim clinical benefit that was not affected by post-treatment therapies. The trial enrolled 1717 chemotherapy-naive mCRPC patients at 207 sites globally [36]. Patients were randomly assigned $1: 1$ to receive enzalutamide $(n=872)$ or placebo $(n=845)$. Treatment continued until the occurrence of unacceptable adverse events (AEs) or confirmed radiographic progression and the initiation of chemotherapy or an investigational agent [36].

At a preplanned interim analysis after 22 months, enzalutamide treatment had significantly decreased the risk of centrally assessed radiographic progression or death by $81 \%$ (HR: 0.19 ; 95\% CI: $0.15-0.23$; p < 0.001 ) and death by 29\% (HR: 0.71; 95\% CI: 0.60-0.84; p < 0.001) versus placebo (Table 4) [36]. The PREVAIL study was unblinded at this point, and eligible patients in the placebo group were offered enzalutamide. Prespecified sensitivity analyses demonstrated the robustness of the Prostate Cancer Clinical Trials Working Group 2 rPFS definition using additional measures of progression, including SREs, initiation of radiotherapy and/or new antineoplastic therapy, and unequivocal clinical progression [48]. There was a positive correlation between rPFS and OS among enzalutamide-treated patients [48]. Enzalutamide continued to provide benefit over placebo after an additional 20 months of follow-up for investigator-assessed rPFS and 9 months of follow-up for OS [49]. In a post hoc analysis, median rPFS was 20.0 months in the enzalutamide group versus 5.4 months in the placebo group (HR: 0.32; $95 \%$ CI: $0.28-0.37 ; \mathrm{p}<0.0001$ ), whereas median OS was 35.3 and 31.3 months, respectively (HR: $0.77 ; 95 \%$ CI: $0.67-0.88 ; \mathrm{p}=0.0002)$ [49]. 
Table 5. Summary of adverse events emergent on treatment with enzalutamide $160 \mathrm{mg}$ /day versus placebo in patients with metastatic castration-resistant prostate cancer (Pfizer Inc., data on file).

\begin{tabular}{|c|c|c|c|c|c|c|}
\hline \multirow[t]{2}{*}{ Event } & \multicolumn{2}{|c|}{ AFFIRM } & \multicolumn{2}{|c|}{ PREVAIL } & \multicolumn{2}{|c|}{ PROSPER } \\
\hline & Enzalutamide $(n=800)$ & Placebo $(n=399)$ & Enzalutamide $(n=871)$ & Placebo $(n=844)$ & Enzalutamide $(\mathrm{n}=930)$ & Placebo $(n=465)$ \\
\hline Median treatment duration, mo & 8.3 & 3.0 & 17.5 & 4.6 & 18.4 & 11.1 \\
\hline Received glucocorticoids, \% & 48 & 46 & 27 & 30 & NR & NR \\
\hline Any AE: & $785(98)$ & $390(98)$ & $844(97)$ & $787(93)$ & $808(87)$ & $360(77)$ \\
\hline - Grade $\geq 3 \mathrm{AE}$ & $362(45)$ & $212(53)$ & $374(43)$ & $313(37)$ & $292(31)$ & $109(23)$ \\
\hline - Any serious $A E$ & $268(34)$ & $154(39)$ & $279(32)$ & $226(27)$ & $226(24)$ & $85(18)$ \\
\hline $\begin{array}{l}\text { - AE leading to trial } \\
\text { discontinuation }\end{array}$ & $61(8)$ & $39(10)$ & $49(6)$ & $51(6)$ & $87(9)$ & $28(6)$ \\
\hline - AE leading to death & $23(3)$ & $14(4)$ & $37(4)$ & $32(4)$ & $32(3)$ & $3(<1)$ \\
\hline \multicolumn{7}{|l|}{ Most common AEs ${ }^{\dagger}$} \\
\hline Fatigue: & $269(34)$ & $116(29)$ & $310(36)$ & $218(26)$ & $303(33)$ & $64(14)$ \\
\hline - Grade $\geq 3$ & $50(6)$ & $29(7)$ & $16(2)$ & $16(2)$ & $27(3)$ & $3(<1)$ \\
\hline Asthenia: & $140(18)$ & $67(17)$ & $113(13)$ & $67(8)$ & $82(9)$ & $28(6)$ \\
\hline - Grade $\geq 3$ & $20(3)$ & $10(3)$ & $11(1)$ & $8(1)$ & $11(1)$ & $1(<1)$ \\
\hline Peripheral edema: & (15) & (13) & $92(11)$ & $69(8)$ & NR & NR \\
\hline - Grade $\geq 3$ & (1) & $(<1)$ & $2(<1)$ & $3(<1)$ & NR & NR \\
\hline Arthralgia: & $(21)$ & (17) & $177(20)$ & $135(16)$ & $78(8)$ & $32(7)$ \\
\hline - Grade $\geq 3$ & (3) & (2) & $12(1)$ & $9(1)$ & $1(<1)$ & $1(<1)$ \\
\hline Back pain: & $(26)$ & (24) & $235(27)$ & $187(22)$ & $73(8)$ & $33(7)$ \\
\hline - Grade $\geq 3$ & $(5)$ & (4) & $22(3)$ & $25(3)$ & $2(<1)$ & $1(<1)$ \\
\hline Musculoskeletal pain: & $109(14)$ & $40(10)$ & NR & NR & NR & NR \\
\hline - Grade $\geq 3$ & $8(1)$ & $1(<1)$ & NR & NR & NR & NR \\
\hline Diarrhea: & $171(21)$ & $70(18)$ & $142(16)$ & $119(14)$ & $91(10)$ & $45(10)$ \\
\hline - Grade $\geq 3$ & $9(1)$ & $1(<1)$ & $2(<1)$ & $3(<1)$ & $3(<1)$ & $2(<1)$ \\
\hline Constipation: & $188(24)$ & $110(28)$ & $193(22)$ & $145(17)$ & $85(9)$ & $32(7)$ \\
\hline - Grade $\geq 3$ & NR & NR & $4(<1)$ & $3(<1)$ & $2(<1)$ & $2(<1)$ \\
\hline Hot flush: & $162(20)$ & $41(10)$ & $157(18)$ & $65(8)$ & $121(13)$ & $36(8)$ \\
\hline - Grade $\geq 3$ & 0 & 0 & $1(<1)$ & 0 & $1(<1)$ & \\
\hline Hypertension: & (6) & (3) & $117(13)$ & $35(4)$ & $111(12)$ & $24(5)$ \\
\hline - Grade $\geq 3$ & (2) & (1) & $59(7)$ & $19(2)$ & $43(5)$ & $10(2)$ \\
\hline Headache: & $93(12)$ & $22(6)$ & $91(10)$ & $59(7)$ & $85(9)$ & $21(5)$ \\
\hline - Grade $\geq 3$ & $6(<1)$ & 0 & $2(<1)$ & $3(<1)$ & $2(<1)$ & 0 \\
\hline Dizziness: & $(10)^{\ddagger}$ & $(8)^{\ddagger}$ & $(11)^{\ddagger}$ & $(7)^{\ddagger}$ & $91(10)$ & $20(4)$ \\
\hline - Grade $\geq 3$ & $(<1)^{\ddagger}$ & $(<1)^{\ddagger}$ & $(<1)^{\ddagger}$ & $0^{\ddagger}$ & $4(<1)$ & 0 \\
\hline Upper respiratory-tract infection: & (11) & (7) & $(16)$ & (11) & NR & NR \\
\hline - Grade $\geq 3$ & 0 & $(<1)$ & 0 & 0 & NR & NR \\
\hline Fall: & (5) & (1) & $101(12)$ & $45(5)$ & $106(11)$ & $19(4)$ \\
\hline - Grade $\geq 3$ & $(<1)$ & 0 & $12(1)$ & $6(1)$ & $12(1)$ & $3(<1)$ \\
\hline Decreased appetite: & NR & NR & $158(18)$ & $136(16)$ & $89(10)$ & $18(4)$ \\
\hline - Grade $\geq 3$ & NR & NR & $2(<1)$ & $6(1)$ & $2(<1)$ & $1(<1)$ \\
\hline Weight loss: & NR & NR & $100(11)$ & $71(8)$ & $55(6)$ & $7(2)$ \\
\hline - Grade $\geq 3$ & NR & NR & $5(1)$ & $2(<1)$ & $2(<1)$ & 0 \\
\hline \multicolumn{7}{|l|}{ AEs of special interest } \\
\hline Any cardiac disorder: & $49(6)$ & $30(8)$ & $88(10)$ & $66(8)$ & NR & NR \\
\hline \multicolumn{7}{|c|}{$\begin{array}{l}\text { Data expressed as } n(\%) \text { unless otherwise specified. } \\
\text { † Occurring in } \geq 10 \% \text { of patients in the enzalutamide group and at an incidence of } \geq 2 \% \text { higher than that in the placebo group in AFFIRM, PREVAIL or PROSPER. } \\
\text { ¥Includes dizziness and vertigo. } \\
\text { §Includes acute myocardial infarction, hemorrhagic cerebrovascular conditions, ischemic CV conditions and heart failure. } \\
\text { I Abnormalities on liver-function testing included hyperbilirubinemia and increased levels of aspartate aminotransferase or alanine aminotransferase. } \\
\text { \#Includes memory impairment, disturbance in attention, cognitive disorders, amnesia, Alzheimer's disease, senile dementia, mental impairment and vascular dementia. } \\
\dagger \dagger \text { Occurred after the data cut-off date. } \\
\text { Data previously presented [20,29,32,36]. } \\
\text { AE: Adverse event; CV: Cerebrovascular; mCRPC: Metastatic castration-resistant prostate cancer. }\end{array}$} \\
\hline
\end{tabular}


Table 5. Summary of adverse events emergent on treatment with enzalutamide $160 \mathrm{mg} /$ day versus placebo in patients with metastatic castration-resistant prostate cancer (Pfizer Inc., data on file) (cont.).

\begin{tabular}{|c|c|c|c|c|c|c|}
\hline \multirow[t]{2}{*}{ Event } & \multicolumn{2}{|c|}{ AFFIRM } & \multicolumn{2}{|c|}{ PREVAIL } & \multicolumn{2}{|c|}{ PROSPER } \\
\hline & Enzalutamide $(n=800)$ & Placebo $(n=399)$ & Enzalutamide $(n=871)$ & Placebo $(n=844)$ & Enzalutamide $(n=930)$ & Placebo $(n=465)$ \\
\hline - Grade $\geq 3$ & $7(1)$ & $8(2)$ & $24(3)$ & $18(2)$ & NR & NR \\
\hline $\begin{array}{l}\text { Major adverse cardiovascular } \\
\text { event: }{ }^{\$}\end{array}$ & NR & NR & $23(3)$ & $16(2)$ & $48(5)$ & $13(3)$ \\
\hline - Grade $\geq 3$ & NR & NR & NR & NR & $34(4)$ & $8(2)$ \\
\hline Atrial fibrillation: & $9(1)$ & $5(1)$ & $16(2)$ & $12(1)$ & NR & NR \\
\hline - Grade $\geq 3$ & NR & NR & $7(1)$ & $2(<1)$ & NR & NR \\
\hline Myocardial infarction: & $2(<1)$ & $2(<1)$ & NR & NR & $7(<1)$ & 0 \\
\hline - Grade $\geq 3$ & $2(<1)$ & $2(<1)$ & $3(<1)$ & $1(<1)$ & NR & NR \\
\hline $\begin{array}{l}\text { Ischemic or hemorrhagic CV } \\
\text { event: }\end{array}$ & (1) & (1) & $12(1)$ & $9(1)$ & $17(<2)$ & $4(<1)$ \\
\hline Hepatic impairment: & NR & NR & $10(1)$ & $8(1)$ & $11(1)$ & $9(2)$ \\
\hline - Grade $\geq 3$ & NR & NR & $3(<1)$ & $3(<1)$ & $5(1)$ & $2(<1)$ \\
\hline $\begin{array}{l}\text { Abnormality on liver function } \\
\text { testing: }\end{array}$ & $8(1)^{\mathbb{I}}$ & $6(2) \mathbb{I}$ & $4(<1)$ & $2(<1)$ & 0 & $1(<1)$ \\
\hline - Grade $\geq 3$ & $3(<1)^{\mathbb{I}}$ & $3(<1) \mathbb{I}$ & NR & NR & 0 & NR \\
\hline Neutropenia: & NR & NR & $13(2)$ & $5(<1)$ & $9(1)$ & $1(<1)$ \\
\hline - Grade $\geq 3$ & (1) & NR & $(<1)$ & $(<1)$ & $5(1)$ & $1(<1)$ \\
\hline Mental impairment disorders:" & NR & NR & $50(6)$ & $13(2)$ & $48(5)$ & 9 (2) \\
\hline - Grade $\geq 3$ & NR & NR & 0 & $2(<1)$ & $1(<1)$ & 0 \\
\hline Convulsion: & $5(<1)$ & 0 & $1(<1)^{\dagger \dagger}$ & $1(<1)$ & $3(<1)$ & 0 \\
\hline - Grade $\geq 3$ & $5(<1)$ & 0 & $1(<1)^{\dagger \dagger}$ & 0 & $2(<1)$ & 0 \\
\hline
\end{tabular}

Data expressed as $n(\%)$ unless otherwise specified

† Occurring in $\geq 10 \%$ of patients in the enzalutamide group and at an incidence of $\geq 2 \%$ higher than that in the placebo group in AFFIRM, PREVAIL or PROSPER.

¥Includes dizziness and vertigo.

§Includes acute myocardial infarction, hemorrhagic cerebrovascular conditions, ischemic CV conditions and heart failure.

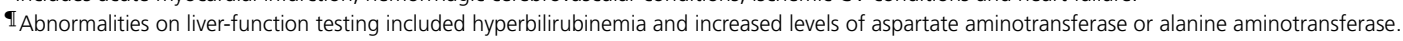

\#Includes memory impairment, disturbance in attention, cognitive disorders, amnesia, Alzheimer's disease, senile dementia, mental impairment and vascular dementia.

†† Occurred after the data cut-off date.

Data previously presented $[20,29,32,36]$.

AE: Adverse event; CV: Cerebrovascular; mCRPC: Metastatic castration-resistant prostate cancer.

Enzalutamide provided statistically significant benefits across all secondary end points (Table 4) and the treatment effects of enzalutamide on rPFS and OS were consistent across all prespecified subgroups [36]. Point estimates for OS were in favor of enzalutamide in patients from North America, Japan and Korea, those with baseline visceral disease (lung and/or liver), and those with a baseline PSA level less than the median ( $49.6 \mathrm{ng} / \mathrm{ml})$ [36,50,51]. Elderly men experienced OS and rPFS benefits with enzalutamide (Table 4) [42].

PREVAIL was unique in that it did not exclude patients with visceral disease. At study baseline, $88 \%$ of patients had nonvisceral disease, $12 \%$ had visceral disease, 50\% had lymph node disease and $85 \%$ had bone metastases. Hence, the PREVAIL population was reflective of the wide range of patients commonly treated by urologists and medical oncologists regarding tumor site and extent of disease [52]. rPFS was greater with enzalutamide than with placebo in patients with nonvisceral disease (HR: 0.18; 95\% CI: 0.14-0.22), visceral disease (HR: 0.28; 95\% CI: 0.16-0.49), low- or high-volume bone disease (HR: 0.16; 95\% CI: 0.11-0.22; HR: 0.22; 95\% CI: 0.16-0.29, respectively), and lymph node only disease (HR: 0.09; 95\% CI: 0.04-0.19) [52]. A related prespecified analysis determined the impact of visceral disease site (lung and liver) on study end points [53]. In patients with liver metastases, enzalutamide improved rPFS (HR: 0.44; 95\% CI: 0.22-0.90) but not OS (HR: 1.04; 95\% CI: 0.571.87). In patients with lung metastases only, the HR for rPFS (0.14; 95\% CI: 0.06-0.36) but not OS (0.59; $95 \%$ 
CI: 0.33-1.06) favored enzalutamide over placebo. Furthermore, the small subset of patients with liver metastases fared worse than those with lung metastases with respect to secondary end points, the latter group exhibiting benefits consistent with those of the full study population [53].

Of interest, 65 of 265 evaluable enzalutamide patients in PREVAIL had a nonrising PSA (defined as $\leq 1.05$-times the PSA level from 3 months earlier) at the time of radiographic progression [54]. Median progression-free survival (PFS) in this nonrising PSA cohort was 8.3 months versus 11.1 months in the rising PSA cohort (HR: 1.68; 95\% CI: 1.26-2.23), while OS was similar between the two groups. These data suggest that reliance on serial PSA measurements alone, at least in the short term, may not be sufficient to identify disease progression in patients receiving enzalutamide and underscores the need for complementary disease-monitoring measures, such as alkaline phosphatase levels and imaging [54].

In recognition of $\mathrm{mCRPC}$ as a heterogeneous disease with respect to clinical characteristics and underlying disease biology, an exploratory analysis from PREVAIL assessed rPFS and OS in men with low PSA $(<10 \mathrm{ng} / \mathrm{ml})$ and either high-volume disease ( $>4$ bone metastases and/or visceral disease) or low-volume disease $(\leq 4$ bone metastases with no visceral disease) [55]. At baseline, 242 patients (14\%) had low PSA, including 110 men with high-volume disease. Enzalutamide decreased the risk of radiographic progression relative to placebo (HR: 0.20; 95\% CI: 0.10-0.42) in patients with low baseline PSA regardless of tumor burden (high-volume disease: HR: 0.17; 95\% CI: 0.06-0.51; low-volume disease: HR: 0.25; 95\% CI: 0.09-0.70). Median OS was not reached in either treatment arm for the subgroup of patients with low baseline PSA. The results provide evidence that patients with low PSA can benefit from AR-targeted therapies even in the setting of high metastatic burden [55].

\section{TERRAIN \& STRIVE: Phase II trials of enzalutamide versus bicalutamide}

TERRAIN was carried out in the same population as PREVAIL, using bicalutamide as an active comparator instead of placebo and a broader definition of the progression end point. The trial randomized 375 asymptomatic or mildly symptomatic, chemotherapy-naive, mCRPC patients at 84 sites across North America and Europe to either enzalutamide $(n=184)$ or bicalutamide $(n=191)$ [34]. Treatment continued until a progression event, an AE necessitating discontinuation, or patient withdrawal. The primary end point was PFS, defined as time from randomization to radiographic disease progression, an SRE, initiation of antineoplastic therapy, or death [34].

Median duration of therapy was longer in the enzalutamide group than in the bicalutamide group (11.7 vs 5.8 months), as was median follow-up time (20.0 vs 16.7 months) [34]. Median PFS was 15.7 months in the enzalutamide group versus 5.8 months in the bicalutamide group (HR: 0.44; 95\% CI: $0.34-0.57$; p $<0.0001$; Table 4).

Enzalutamide-treated patients also showed improvements in secondary end point measures, including investigator-assessed PFS objective response, time to PSA progression, PSA response by week 13, best PSA response, and time to a 50\% PSA decline (Table 4). The superior efficacy of enzalutamide over bicalutamide regarding PFS was detected across all prespecified subgroups, including age, geographical location, baseline Eastern Cooperative Oncology Group Performance Status (ECOG PS), baseline PSA, whether ADT was initiated before or after diagnosis of metastatic disease, and previous use of antiandrogens [34].

Although similar to TERRAIN in design, STRIVE was the first randomized trial to enroll men with nmCRPC $(\mathrm{n}=139)$ as well as mCRPC $(\mathrm{n}=257)$ [35]. Patients received enzalutamide $(\mathrm{n}=198)$ or bicalutamide $50 \mathrm{mg} / \mathrm{day}$ $(\mathrm{n}=198)$ until confirmed PSA or radiographic progression or until an AE that would lead to undue risk if dosing had continued [35].

Enzalutamide treatment extended PFS (primary end point) and reduced the risk of progression or death by $76 \%$ compared with bicalutamide (Table 4). Enzalutamide significantly improved all key secondary end points, including rPFS in metastatic patients (not yet reached [NYR] vs 8.3 months; HR: 0.32; 95\% CI: 0.21-0.50; $\mathrm{p}<0.001$ ). Prolongation in PFS by enzalutamide was consistently observed in both the nmCRPC and mCRPC subgroups, and in groups by baseline age, ECOG PS, Gleason score, use of bone-targeting agents, and PSA, LDH, and hemoglobin levels above versus below median values. Patients with nmCRPC treated with enzalutamide fared better in disease stability and PSA progression and responses than those with mCRPC. STRIVE and TERRAIN demonstrated the superiority of enzalutamide over bicalutamide and provided clinical evidence for the improved AR binding characteristics of enzalutamide.

\section{Nonmetastatic disease: enzalutamide versus placebo}

Until 2018, there was no approved therapy for patients with nmCRPC. The Phase III PROSPER trial was, in part, informed by the efficacy seen with enzalutamide in the subgroup of patients with nmCRPC in STRIVE [20]. 
PROSPER patients had rapidly rising PSA values and, thus, were at high risk for progression. Median baseline PSA level was $>10 \mathrm{ng} / \mathrm{ml}$, and median PSA doubling time was 3.7 months (77\% had PSA doubling time of $<6$ months), all of which predispose to a high risk of metastases. Patients were stratified according to PSA doubling time ( $<6$ vs $\geq 6$ months) and previous or current use of a bone-targeting agent at baseline prior to random assignment (2:1) to either enzalutamide $(n=933)$ or placebo $(n=468)$. Treatment continued until radiographic progression assessed by central independent blinded radiographic review, clinical progression or AEs [20].

Median duration of treatment was 18.4 months for enzalutamide and 11.1 months for placebo [20]. Median MFS, the primary end point, was 36.6 months with enzalutamide treatment versus 14.7 months with placebo. Enzalutamide treatment resulted in a $71 \%$ reduction in the risk of radiographic progression or death (HR: 0.29; 95\% CI: $0.24-0.35 ; \mathrm{p}<0.001)$. Patients receiving enzalutamide had longer median times to PSA progression and first use of a subsequent antineoplastic therapy, and more had PSA responses $\geq 50 \%$ (Table 4 ) than those receiving placebo. At the first interim analysis of OS, deaths were reported for $11 \%(\mathrm{n}=103)$ of patients receiving enzalutamide and $13 \%(\mathrm{n}=62)$ receiving placebo [20].

The enzalutamide treatment effect was consistent across all prespecified subgroups, including those defined by PSA doubling time ( $<6$ vs $\geq 6$ months), age, geographic region (North America vs Europe vs rest of world), ECOG PS, Gleason score, use of bone-targeting agents, and PSA, LDH and hemoglobin levels above versus below their median values [20].

\section{Patient-reported outcomes}

The arrival of life-extending noncytotoxic therapies for PC has led to an increased interest in the use of PRO measures in clinical trials. Formal evaluation of patient experiences and perceptions provides a comprehensive appreciation of efficacy and safety and, importantly, these experiences may have significant associations with disease progression and survival $[19,56]$. The most widely used PRO measure in PC is the Functional Assessment of Cancer Therapy-Prostate (FACT-P). In AFFIRM, enzalutamide treatment significantly improved function and QoL; prespecified analyses showed extended times to first SRE, pain progression, and deterioration in FACT-P global score (Tables $4 \& 6$ ) [57]. The lower risk of first SRE and pain progression with enzalutamide versus placebo was evident irrespective of bisphosphonate or corticosteroid use at baseline [57]. In addition, $45 \%$ of evaluable patients $(22 / 49)$ in the enzalutamide group versus $7 \%(1 / 15)$ in the placebo group reported pain palliation at week 13 (difference 38.2\%, 95\% CI: 19.4-57.0; $\mathrm{p}=0.0079$ ) and a higher proportion of patients in the enzalutamide group reported clinically significant improvements in FACT-P global score ( 42 vs $15 \%, \mathrm{p}<0.0001$ vs placebo) [57]. A subsequent longitudinal analysis of AFFIRM revealed that enzalutamide treatment was associated with stabilization of the FACT-P global score, whereas HRQoL measures deteriorated steadily with placebo [58].

The PREVAIL patient population reported mild HRQoL impairment on the FACT-P at baseline [59]. Despite this, efficacy and acceptable tolerability translated into improved health status for enzalutamide relative to placebo, based on mean change-from-baseline scores, times to clinically significant deterioration, and FACT-P and EQ5D responses (Table 6). The changes from baseline, median time to deterioration and improvement in FACT-P PC subscale score also favored enzalutamide over placebo, as did median times to deterioration in all other FACT-P subscale scores [59]. In a post hoc analysis of PREVAIL based on the generic HRQoL EQ-5D scale, enzalutamide treatment delayed HRQoL deterioration and had beneficial effects on several HRQoL domains, including pain/discomfort and the proportion of patients in full health, relative to placebo [61]. The effect of enzalutamide on most Brief Pain Inventory Short Form (BPI-SF) pain metrics was favorable relative to placebo, with the caveat that the PREVAIL population was largely asymptomatic at baseline [59].

In a pooled analysis of AFFIRM and PREVAIL, SREs were associated with clinically meaningful functional declines in the daily lives of patients with mCRPC [62]. Of the SREs, spinal cord compression induced the largest reductions in FACT-P total scores and the largest effect sizes across HRQoL metrics.

There were greater improvements in HRQoL associated with enzalutamide than bicalutamide in TERRAIN and STRIVE (Table 6) $[34,35,60]$. At week 61 in TERRAIN, time to FACT-P total score deterioration was longer and rates of improvement in various FACT-P scores were higher for patients receiving enzalutamide than for those receiving bicalutamide $[34,60]$. The BPI-SF scores increased in both groups, with no between-group differences in time-to-pain progression [60]. Thus, time to documented decline in QoL (based on FACT-P scores) was approximately 5 months longer with enzalutamide than placebo in men with mCRPC, although not significantly different in men with nmCRPC. When compared with bicalutamide, time to QoL deterioration with enzalutamide was significantly longer in TERRAIN but not in STRIVE. 


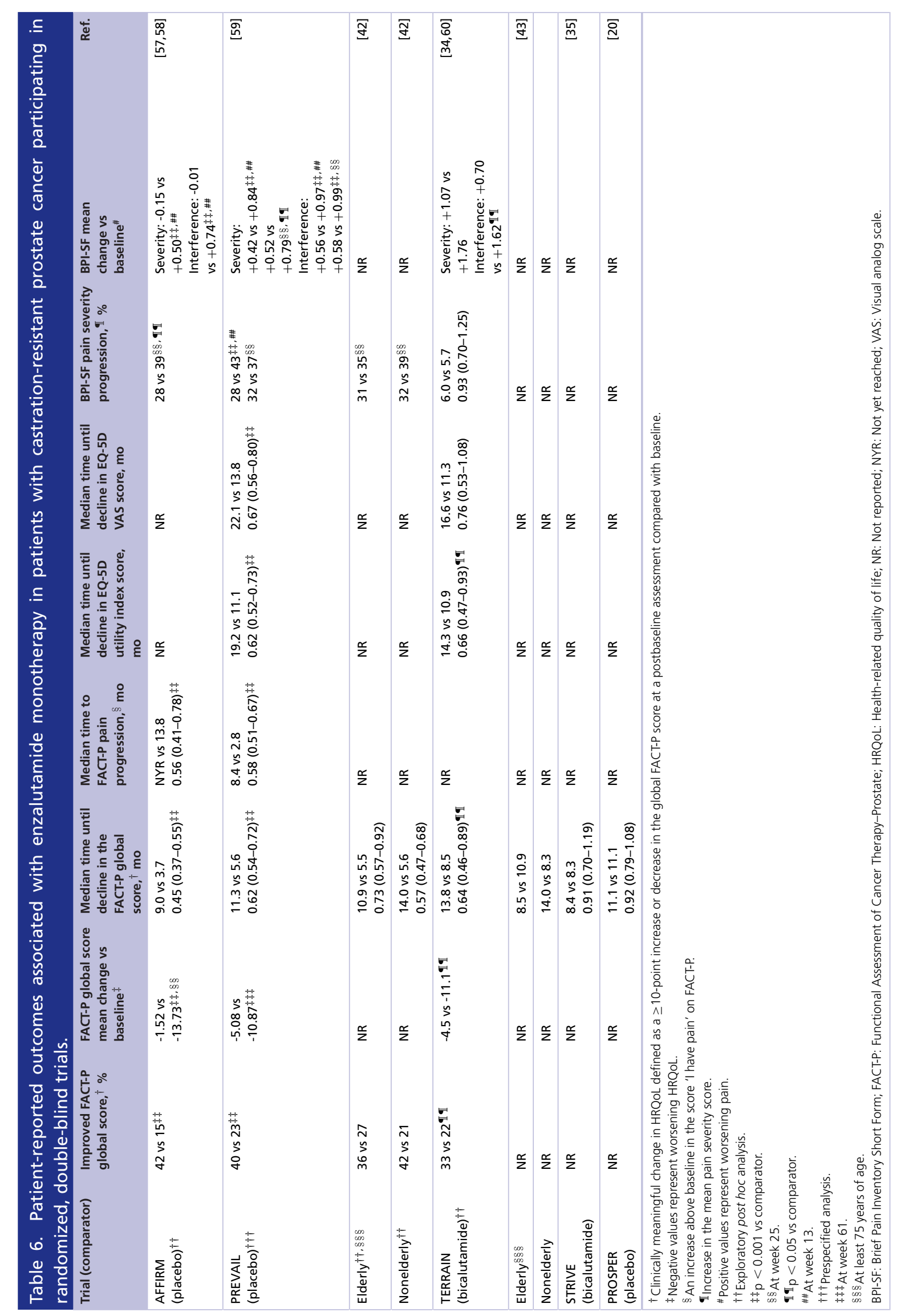


Safety \& tolerability

Men with PC are now surviving longer, such that AEs can affect present and future function and overlap with the AEs of subsequent or combination therapy. While the safety profiles of newer agents differ greatly from chemotherapy, class-specific AEs can drive treatment decisions. Fatigue, hypertension, cognitive impairment, falls and fracture have been seen with enzalutamide treatment (Table 5); preliminary studies of new AR-targeting therapies suggest that several of these AEs may be a class effect.

Phase III placebo-controlled trials

In AFFIRM, incidences of grade 3 or higher AEs and serious AEs were slightly lower in the enzalutamide group than the placebo group (grade 3 or higher AEs: 45 vs 53\%; serious AEs: 34 vs 39\%) [32]. Conversely, AE incidences were slightly higher with enzalutamide than placebo in PREVAIL (grade 3 or 4 AEs: 43 vs 37\%; serious AEs: 32 vs $27 \%$ ) [36]. The AEs occurring more frequently with enzalutamide than placebo in PREVAIL (after adjustment for length of exposure) were hot flush (14 vs 12 events per 100 patient-years), hypertension (11 vs seven events per 100 patient-years) and falls (11 vs nine events per 100 patient-years) [36]. Median latency to a first grade $\geq 3 \mathrm{AE}$ was longer in the enzalutamide groups than placebo groups in both AFFIRM (12.6 vs 4.2 months) and PREVAIL (22.3 vs 13.3 months) [32,36]. Disease progression was the most common AE leading to death in AFFIRM and PREVAIL [32,36]. In AFFIRM, a thorough QT study reported that enzalutamide did not prolong the QT interval [32]. Elderly patients experienced more grade $\geq 3$ fatigue than younger patients in AFFIRM (9 vs 5\%) [41], and in PREVAIL [42], there was an overall higher incidence of falls among elderly patients receiving enzalutamide than those receiving placebo (19 vs $8 \%$ ) [42].

Among men with nmCRPC in PROSPER, grade 3 or higher AEs (31 vs 23\%) and serious AEs (24 vs 18\%) occurred more frequently with enzalutamide treatment than placebo [20]. Of note, patients were on treatment for a longer time in the enzalutamide arm than in the placebo arm to evaluate AEs. The median reporting period for AEs was 18.0 months for enzalutamide versus 11.1 months for placebo and AEs were reported up to 30 days after the last dose. Hypertension was more common in patients treated with enzalutamide (12 vs 5\% with placebo) [29]. The most common AEs leading to death in PROSPER were cardiac events, which occurred in nine (1\%) of 930 patients receiving enzalutamide and two $(0.4 \%)$ of 465 patients receiving placebo [20].

\section{Phase IV safety results}

Results from a Phase IV, open-label, expanded-access program across North America has provided real-world AE data from a cohort of heavily pretreated men with mCRPC. In this population $(n=507)$, enzalutamide was well tolerated with a safety profile largely consistent with that of AFFIRM [32,63]. Specifically, 88\% of patients experienced AEs, $45 \%$ of which were a maximum of grade $1-2$. Fatigue (39\%), nausea $(23 \%)$ and anorexia (15\%) were the most commonly reported AEs. Seizure was reported in four patients $(0.8 \%)$. The most commonly reported event leading to death was progression of metastatic PC (8\%) [63]. Despite low performance status and a median of five prior antineoplastic therapies in this cohort, safety data were consistent with those in clinical trials, and no additional safety signals were identified.

\section{Safety summary}

When summarized across randomized trials $(\mathrm{n}=2784)$ the most common AEs (in $>10 \%$ of patients) that occurred more frequently with enzalutamide treatment $(>2 \%$ over placebo $[n=1708])$ were asthenia/fatigue, decreased appetite, hot flush, arthralgia, dizziness/vertigo, hypertension, headache and decreased weight [29]. Falls (10 vs $4 \%$ ) and fractures (8 vs 3\%) were also more frequent among patients receiving enzalutamide than those receiving placebo $[20,29,32,36]$.

Warnings and precautions on the enzalutamide product label include seizures, posterior reversible encephalopathy syndrome, hypersensitivity, ischemic heart disease, falls and fractures, and embryo-fetal toxicity [64]. In clinical trials that excluded patients with a predisposition to seizure, seizure occurred in $0.5 \%$ of patients receiving enzalutamide, $0.1 \%$ receiving placebo and $0.3 \%$ receiving bicalutamide. In the Phase IV UPWARD study (NCT01977651) of mCRPC patients with at least one risk factor for seizure, four of 366 evaluable patients $(1.1 \%)$ had one or more confirmed seizure within 4 months of enzalutamide initiation and three $(0.8 \%)$ additional patients experienced a seizure within 4 months following the 4-month study period [65]. Incidence of confirmed seizure was 2.6 per 100 patient-years, which compares favorably with an estimated seizure rate of 2.8 per 100 patient-years among men with $\mathrm{mCRPC}$ and seizure risk factors without enzalutamide exposure [65]. 


\begin{tabular}{|c|c|c|c|c|c|}
\hline Study & Type of patients & Prognostic variable & Definition & $\begin{array}{l}\text { Associated clinical } \\
\text { outcome(s) }\end{array}$ & Ref. \\
\hline AFFIRM & $\begin{array}{l}\text { - Metastatic } \\
\text { - Prior chemotherapy } \\
\text { - ECOG PS: 0-5 } \\
\text { - BPI-SF Q3: } 0-10\end{array}$ & PSA decline & $\begin{array}{l}\text { PSA decline of any amount, } \geq 30 \% \text {, and } \\
\geq 50 \% \text { in the first } 90 \text { days of } \\
\text { enzalutamide treatment }\end{array}$ & $\begin{array}{l}- \text { OS } \\
\text { - rPFS } \\
\text { - PSA PFS } \\
\text { - Pain response }\end{array}$ & [71] \\
\hline \multirow[t]{10}{*}{ PREVAIL } & $\begin{array}{l}\text { - Metastatic } \\
\text { - Chemotherapy-naive } \\
\text { - Progressed on ADT } \\
\text { - Asymptomatic/mildly } \\
\text { symptomatic } \\
\text { - ECOG PS: } 0-1 \\
\text { - Visceral disease allowed }\end{array}$ & PSA decline & $\begin{array}{l}\text { PSA decline of } \geq 30, \geq 50 \text { and } \geq 90 \% \\
\text { from baseline to week } 12\end{array}$ & $\begin{array}{l}\text { - rPFS } \\
\text { - OS } \\
\text { - Time to PSA progression } \\
\text { - FACT-P decline } \\
\text { - BPI-SF pain severity } \\
\text { progression }\end{array}$ & {$[72,73]$} \\
\hline & & Albumin & Continuous, per point rise in $\mu \mathrm{g} / \mathrm{dl}$ & - OS & \\
\hline & & Alkaline phosphatase & $<U L N$ vs $\geq U L N$ & & \\
\hline & & Hemoglobin & Continuous, $\mathrm{g} / \mathrm{dl}$ & & \\
\hline & & LDH & $<\mathrm{ULN}$ vs $\geq 1 \mathrm{X}$ ULN & & \\
\hline & & Neutrophil-to-lymphocyte ratio & $<2.5$ vs $\geq 2.5$ & & \\
\hline & & Number of bone metastases & $<10$ vs $\geq 10$ & & \\
\hline & & Pain score & $0-1$ vs $\geq 2$ (linear scale) & & \\
\hline & & Pattern of spread & $\begin{array}{l}\text { No liver metastases vs any liver } \\
\text { metastases }\end{array}$ & & \\
\hline & & $\begin{array}{l}\text { Time from diagnosis to } \\
\text { randomization }\end{array}$ & Continuous, months & & \\
\hline \multicolumn{6}{|c|}{$\begin{array}{l}\text { ADT: Androgen deprivation therapy; BPI-SF: Brief Pain Inventory-Short Form; ECOG PS: Eastern Cooperative Oncology Group Performance Status; FACT-P: Functional Assessment of } \\
\text { Cancer Therapy-Prostate; LDH: Lactate dehydrogenase; OS: Overall survival; PFS: Progression-free survival; PSA: Prostate-specific antigen; rPFS: Radiographic PFS; ULN: Upper limit of } \\
\text { normal. }\end{array}$} \\
\hline
\end{tabular}

\section{Prognostic associations in enzalutamide trials}

There are well-established clinical factors that are prognostic for OS in mCRPC [66], and response to therapy can also contribute meaningfully to prognosis [67-69]. As PSA decline has been strongly associated with OS in PC trials of agents with varying targets, the association between direct AR inhibition with enzalutamide, PSA and survival outcomes has been particularly informative [67-70].

Men treated with enzalutamide in AFFIRM had greater OS and higher rates of PSA decline than those receiving placebo [71]. When evaluated by magnitude of decline, PSA declines of any amount, $\geq 30$ and $\geq 50 \%$ in the first 90 days of enzalutamide treatment were more tightly associated with OS, rPFS, PSA PFS ( $<<0.001)$, and pain response $(p<0.026)$ than a PSA increase or no decline (Table 7) [71]. Surrogacy of PSA decline for improved OS was not fully established, although a lack of PSA decline by week 12 following enzalutamide treatment was an indicator of poor prognosis [71].

Prognostic association between the magnitude of PSA decline from baseline to week 12 and clinical outcomes in PREVAIL have also been evaluated post hoc, with declines of $\geq 30, \geq 50$ and $\geq 90 \%$ meeting surrogacy criteria for rPFS, OS, and time to PSA progression, FACT-P decline and BPI-SF pain severity progression [72]. In addition to PSA, ten other prognostic factors for OS were identified in PREVAIL (i.e., albumin, alkaline phosphatase, hemoglobin, LDH, neutrophil-to-lymphocyte ratio, number of bone metastases, pain score, pattern of spread, time from diagnosis to randomization and enzalutamide treatment), which have been incorporated into a validated model to identify subsets of men with different predicted survival ranges [73].

Although much has been learned in recent years about prognostic factors from clinical trial data, it is research into predictive biomarkers (e.g., from circulating tumor cells, cell-free DNA or microRNA) that promises the next major treatment advance.

\section{Mechanisms of resistance with enzalutamide}

The identification of the AR splice variant, AR-V7, has been a meaningful first step in defining mechanisms of resistance with AR-targeting therapies and tailoring treatment. Among patients treated with enzalutamide, approximately $25 \%$ show primary resistance. Several other potential resistance mechanisms have been identified, including AR chromosome copy number amplification, mutations in the AR ligand-binding domain, altered steroidogenesis, overexpression or upregulation of the glucocorticoid receptor, epithelial-to-mesenchymal transition, neuroendocrine 
transformation, activation of other signaling pathways, autophagy and immune system activation [74-79]. Primary resistance to enzalutamide has not been associated with AR agonism based on evidence from a single-center observational study. The occurrence of antiandrogen withdrawal syndrome was uncommon (5/47) and of short duration following enzalutamide discontinuation [80]. Among the five patients who experienced antiandrogen withdrawal syndrome, PSA responses were 84, 32, 17, 15 and 15\%. No patient, disease or treatment characteristic differed between patients with and without antiandrogen withdrawal syndrome [80].

The two-period, Phase IV PLATO trial (NCT01995513) was designed to investigate ligand-binding domain reactivation of AR signaling as a mechanism of resistance [40]. It was hypothesized that raised androgens despite enzalutamide treatment could be overcome by combination with abiraterone, an androgen biosynthesis inhibitor. Men were treated with enzalutamide until PSA progression and then randomized to abiraterone $(1000 \mathrm{mg}$ daily and prednisone $5 \mathrm{mg}$ twice daily) with continued enzalutamide or with placebo. Median PFS was 5.7 months in the enzalutamide combination group and 5.6 months in the placebo combination group (HR: 0.83 ; $95 \%$ CI: $0.61-1.12 ; \mathrm{p}=0.22$ ), demonstrating that sensitivity to AR antagonism by enzalutamide is not reinitiated by concomitant abiraterone exposure [40].

Limited retrospective sequencing data suggest that there is no difference in efficacy with respect to using enzalutamide after abiraterone or abiraterone after enzalutamide, and the presence of cross-resistance between these agents remains an area of study [81].

\section{Ongoing enzalutamide studies in CRPC}

Ongoing trials with enzalutamide continue to evaluate efficacy with combination therapy and sequencing of regimens. An open-label Phase III study (NCT01949337) has been underway in patients with mCRPC randomized to enzalutamide with or without abiraterone and prednisone to assess the possibility of synergy with first-line treatment in chemotherapy-naive men. A Phase III trial of second-line treatment (PRESIDE; NCT02288247) is currently evaluating PFS with enzalutamide versus docetaxel in chemotherapy-naive patients. Men in this trial receive open-label treatment with enzalutamide, which upon progression is followed by randomization to continued enzalutamide plus docetaxel or to docetaxel plus placebo. Responding to the need for information regarding PC management, real-world data on prescribing patterns, HRQoL, clinical outcomes and healthcare utilization are being collected in the prospective, observational Treatment Registry for Outcomes in CRPC Patients (TRUMPET; NCT02380274) [82].

\section{Conclusion}

Increasing PSA levels are a hallmark of progressive disease in PC. By inhibiting AR function in the CRPC state, enzalutamide suppresses circulating PSA levels.

Treatment with enzalutamide has resulted in a high level of disease control in different disease settings and in subgroups of patients with mCRPC and nmCRPC across several landmark clinical trials. Prolonged times to key events, such as OS, rPFS, PFS, metastasis, PSA progression, first SRE and cytotoxic chemotherapy initiation, were accompanied by substantial PSA and objective responses among those with soft-tissue or widespread bone disease (in AFFIRM and PREVAIL). Results of PROSPER have already changed the way nmCRPC is treated, where watchful waiting had previously been a standard approach.

Overall, enzalutamide was well tolerated in younger and elderly patients, with a safety profile that was generally consistent across diverse clinical trial populations. All men treated with enzalutamide, particularly the elderly, require counseling on the risk of falls. Although safety findings do not currently indicate increased risk of seizure, enzalutamide should be used with caution among patients with predisposing factors. As enzalutamide becomes available for more men in the earlier stages of CRPC, further safety analyses may be required to assess whether specific subgroups of patients are at higher risk for certain AEs.

PC is a heterogeneous and complex disease historically classified by PSA progression, the presence of metastases and/or history of chemotherapy. The effects of enzalutamide on PSA progression may warrant a re-evaluation of the classifications of disease states within CRPC to incorporate sensitivity and resistance to AR inhibition. It is noteworthy mechanistically that median times to PSA progression increased with treatment at earlier stages of CRPC. This pattern underscores the relationship between the pathology of AR signaling and PC progression.

As an inhibitor of AR signaling with an extensive clinical trial program, enzalutamide has made a unique contribution to the current understanding of PC treatment. Its development has been carried out in parallel with four other agents (abiraterone, cabazitaxel, sipuleucel- $T$ and radium-223), all with different therapeutic targets that have shown survival benefits. Two other novel AR inhibitors, apalutamide and darolutamide, are in active 
clinical development. Results with apalutamide in the SPARTAN trial were presented at nearly the same time as PROSPER and have demonstrated very similar results with a similar reduction in the risk of MFS in patients with nmCRPC [21]. Thus, even within this group of therapies and also considering the rapidly escalating advances in the understanding of DNA repair defects and in immuno-oncology, the present challenge for clinicians is to maximize currently available evidence to personalize the treatment of patients with CRPC and hopefully to improve upon already excellent results with better patient selection and combination therapies.

\section{Future perspective}

The goal of treatment in CRPC is to obtain a cure. Treatment with enzalutamide has prolonged OS and PFS in a wide range of CRPC patient populations; however, challenges remain. The optimal sequencing of novel hormonal therapies for CRPC is still unclear, and more work is necessary to better understand the mechanisms of resistance. Genomic profiling of patients with PC has become standard in most clinical trials and will help identify which patients will benefit from a particular therapy. Combination studies with poly (ADP-ribose) polymerase inhibitors (NCT03395197) and anti-PD-1 therapies (NCT03338790) are also ongoing in CRPC with the aim of further improving outcomes. Looking beyond CRPC, enzalutamide is also being evaluated in hormone-sensitive PC in the ongoing ARCHES trial in the metastatic setting and EMBARK (NCT02319837) in the nonmetastatic setting.

Executive summary

Background

- Disease progression in castration-resistant prostate cancer (CRPC) remains dependent on androgen receptor (AR) signaling.

Enzalutamide mechanism of action

- Enzalutamide is a nonsteroidal antiandrogen that, upon binding the AR, inhibits AR nuclear translocation, DNA binding and coactivator recruitment.

- Enzalutamide represents a molecular advance over first-generation antiandrogens because AR antagonism is retained in the CRPC setting; this is not the case with bicalutamide and flutamide, which convert to partial agonists when the AR is overexpressed.

Clinical efficacy

- Results of Phase III studies have been reported in the metastatic castration-resistant prostate cancer setting after (AFFIRM) and before (PREVAIL) docetaxel chemotherapy.

- In AFFIRM, median overall survival (OS; primary end point) was longer in the enzalutamide group than placebo group (18.4 vs 13.6 months, hazard ratio [HR]: 0.63; $p<0.001$ ).

- In PREVAIL, coprimary end points of radiographic progression-free survival or death (not yet reached vs 3.9 months, HR: $0.19 ; 95 \% \mathrm{Cl}: 0.15-0.23 ; \mathrm{p}<0.001$ ) and OS (32.4 vs 30.2 months, HR: $0.71 ; 95 \% \mathrm{Cl}: 0.60-0.84$; $\mathrm{p}<0.001$ ) were longer in the enzalutamide group than the placebo group.

- Two Phase II studies (TERRAIN and STRIVE) in the CRPC setting were conducted to evaluate enzalutamide versus bicalutamide, given the high prevalence of bicalutamide use in clinical practice.

- Progression-free survival or death, the primary end point in both trials, was significantly extended with enzalutamide treatment relative to bicalutamide treatment (TERRAIN: 15.7 vs 5.8 months, HR: $0.44 ; 95 \% \mathrm{Cl}$ : 0.34-0.57; $p<0.0001$; STRIVE: 19.4 vs 5.7 months, HR: $0.24 ; 95 \% \mathrm{Cl}: 0.18-0.32 ; \mathrm{p}<0.001$ ).

- In the PROSPER study of nonmetastatic castration-resistant prostate cancer men, median metastasis-free survival was 36.6 months in the enzalutamide group versus 14.7 months in the placebo group (HR: $0.29 ; 95 \% \mathrm{Cl}$ : $0.24-0.35 ; p<0.001)$. There was a $71 \%$ reduction in the risk of metastasis-free survival in high-risk patients treated with enzalutamide.

- In all five clinical trials, enzalutamide improved key secondary end points, such as indicators of response (soft tissue and prostate-specific antigen) and indicators of progression (times to prostate-specific antigen decline, first skeletal-related event, chemotherapy, health-related quality of life and pain).

- The efficacy of enzalutamide was apparent in virtually all subgroups analyzed, including the elderly.

Safety

- Enzalutamide was associated with an increased incidence of asthenia/fatigue, decreased appetite, hot flush, arthralgia, dizziness/vertigo, hypertension, headache, decreased weight and falls.

Conclusion

- Enzalutamide represents a major advance in the treatment of CRPC.

- Time to events and response rates were superior to placebo and bicalutamide in the CRPC setting.

- Enzalutamide was well tolerated in clinical trials, confirmed by favorable health-related quality of life scores among patients on treatment. 


\section{Acknowledgements}

The author was fully responsible for all content and editorial decisions.

Financial \& competing interests disclosure

The author has received research funding from Cougar Biotechnology (now Janssen Oncology), Medivation, Pfizer and Clovis, and honoraria from Janssen, Astellas, Sanofi, Novartis, Bayer and AstraZeneca. The author has no other relevant affiliation or financial involvement with any organization or entity with a financial interest in or financial conflict with the subject matter or materials discussed in the manuscript apart from those disclosed.

Medical writing and editorial support were provided by M Darkes, S Vadasz and S Davis of Ashfield Healthcare Communications and funded by Pfizer Inc. and Astellas Pharma, Inc., the co-developers of enzalutamide.

\section{References}

Papers of special note have been highlighted as: $\bullet$ of interest; $\bullet \bullet$ of considerable interest

1. Scher HI, Halabi S, Tannock I et al. Design and end points of clinical trials for patients with progressive prostate cancer and castrate levels of testosterone: recommendations of the Prostate Cancer Clinical Trials Working Group. J. Clin. Oncol. 26(7), 1148-1159 (2008).

2. Scher HI, Sawyers CL. Biology of progressive, castration-resistant prostate cancer: directed therapies targeting the androgen-receptor signaling axis. J. Clin. Oncol. 23(32), 8253-8261 (2005).

3. Holzbeierlein J, Lal P, LaTulippe E et al. Gene expression analysis of human prostate carcinoma during hormonal therapy identifies androgen-responsive genes and mechanisms of therapy resistance. Am. J. Pathol. 164(1), 217-227 (2004).

4. Visakorpi T, Hyytinen E, Koivisto P et al. In vivo amplification of the androgen receptor gene and progression of human prostate cancer. Nat. Genet. 9(4), 401-406 (1995).

5. Chen CD, Welsbie DS, Tran C et al. Molecular determinants of resistance to antiandrogen therapy. Nat. Med. 10(1), 33-39 (2004).

6. Waltering KK, Urbanucci A, Visakorpi T. Androgen receptor (AR) aberrations in castration-resistant prostate cancer. Mol. Cell. Endocrinol. 360(1-2), 38-43 (2012).

7. Kelly WK, Slovin S, Scher HI. Steroid hormone withdrawal syndromes. Pathophysiology and clinical significance. Urol. Clin. North Am. 24(2), 421-431 (1997).

8. Paul R, Breul J. Antiandrogen withdrawal syndrome associated with prostate cancer therapies: incidence and clinical significance. Drug Saf. 23(5), 381-390 (2000).

9. Montgomery RB, Mostaghel EA, Vessella R et al. Maintenance of intratumoral androgens in metastatic prostate cancer: a mechanism for castration-resistant tumor growth. Cancer Res. 68(11), 4447-4454 (2008).

10. Attard G, de Bono JS. Translating scientific advancement into clinical benefit for castration-resistant prostate cancer patients. Clin. Cancer Res. 17(12), 3867-3875 (2011).

11. Massard C, Fizazi K. Targeting continued androgen receptor signaling in prostate cancer. Clin. Cancer Res. 17(12), 3876-3883 (2011).

12. Tran C, Ouk S, Clegg NJ et al. Development of a second-generation antiandrogen for treatment of advanced prostate cancer. Science 324(5928), 787-790 (2009).

- Describes the development of enzalutamide in Phase I/II trials.

13. Bray F, Ferlay J, Soerjomataram I, Siegel RL, Torre LA, Jemal A. Global cancer statistics 2018: GLOBOCAN estimates of incidence and mortality worldwide for 36 cancers in 185 countries. CA Cancer J. Clin. 68(6), 394-424 (2018).

14. Siegel RL, Miller KD, Jemal A. Cancer statistics, 2018. CA Cancer J. Clin. 68(1), 7-30 (2018).

15. Knudsen KE, Scher HI. Starving the addiction: new opportunities for durable suppression of AR signaling in prostate cancer. Clin. Cancer Res. 15(15), 4792-4798 (2009).

16. Kirby M, Hirst C, Crawford ED. Characterising the castration-resistant prostate cancer population: a systematic review. Int. J. Clin. Pract. 65(11), 1180-1192 (2011).

17. Scosyrev E, Messing EM, Mohile S, Golijanin D, Wu G. Prostate cancer in the elderly: frequency of advanced disease at presentation and disease-specific mortality. Cancer 118(12), 3062-3070 (2012).

18. Tannock IF, de Wit R, Berry WR et al. Docetaxel plus prednisone or mitoxantrone plus prednisone for advanced prostate cancer. $N$. Engl. J. Med. 351(15), 1502-1512 (2004).

19. Nussbaum N, George DJ, Abernethy AP et al. Patient experience in the treatment of metastatic castration-resistant prostate cancer: state of the science. Prostate Cancer Prostatic Dis. 19(2), 111-121 (2016).

20. Hussain M, Fizazi K, Saad F et al. Enzalutamide in men with nonmetastatic, castration-resistant prostate cancer. N. Engl. J. Med. 378(26), 2465-2474 (2018).

-. Primary reporting of the Phase III PROSPER trial showing the efficacy and safety of enzalutamide among nonmetastatic castration-resistant prostate cancer patients. 
21. Smith MR, Saad F, Chowdhury S et al. Apalutamide treatment and metastasis-free survival in prostate cancer. N. Engl. J. Med. 378(15), 1408-1418 (2018).

-• Primary reporting of the SPARTAN trial of the androgen receptor inhibitor, apalutamide.

22. Beaver JA, Kluetz PG, Pazdur R. Metastasis-free survival - a new end point in prostate cancer trials. N. Engl. J. Med. 378(26), 2458-2460 (2018).

- Editorial on the value and challenges of metastasis-free survival as a clinical trial end point.

23. Scher HI, Morris MJ, Stadler WM et al. Trial design and objectives for castration-resistant prostate cancer: updated recommendations from the Prostate Cancer Clinical Trials Working Group 3. J. Clin. Oncol. 34(12), 1402-1418 (2016).

24. Jung ME, Ouk S, Yoo D et al. Structure-activity relationship for thiohydantoin androgen receptor antagonists for castration-resistant prostate cancer (CRPC). J. Med. Chem. 53(7), 2779-2796 (2010).

25. Guerrero J, Alfaro IE, Gomez F, Protter AA, Bernales S. Enzalutamide, an androgen receptor signaling inhibitor, induces tumor regression in a mouse model of castration-resistant prostate cancer. Prostate 73(12), 1291-1305 (2013).

26. Rodriguez-Vida A, Galazi M, Rudman S, Chowdhury S, Sternberg CN. Enzalutamide for the treatment of metastatic castration-resistant prostate cancer. Drug Des. Devel. Ther. 9, 3325-3339 (2015).

27. Gibbons JA, Ouatas T, Krauwinkel W et al. Clinical pharmacokinetic studies of enzalutamide. Clin. Pharmacokinet. 54(10), 1043-1055 (2015).

28. Scher HI, Beer TM, Higano CS et al. Antitumour activity of MDV3100 in castration-resistant prostate cancer: a Phase I-II study. Lancet 375(9724), 1437-1446 (2010).

29. XTANDI (enzalutamide) capsules for oral use. Prescribing information. Astellas Pharma Inc. (2012) www.astellas.us/docs/12A005-ENZ-WPI.PDF

30. Center For Drug Evaluation And Research. Application number: 203415Orig1s000. Clinical pharmacology and biopharmaceutics review(s). NDA 20,3415 Review - Enzalutamide. (2018) www.accessdata.fda.gov/drugsatfda_docs/nda/2012/203415Orig1s000ClinPharmR.pdf

31. Gibbons JA, de Vries M, Krauwinkel W et al. Pharmacokinetic drug interaction studies with enzalutamide. Clin. Pharmacokinet. 54(10), 1057-1069 (2015).

32. Scher HI, Fizazi K, Saad F et al. Increased survival with enzalutamide in prostate cancer after chemotherapy. N. Engl. J. Med. 367(13), 1187-1197 (2012).

- Primary reporting of the Phase III AFFIRM trial showing the safety and efficacy of enzalutamide among patients with castration-resistant prostate cancer previously treated with docetaxel-based chemotherapy.

33. Lissbrant IF, Garmo H, Widmark A, Stattin P. Population-based study on use of chemotherapy in men with castration resistant prostate cancer. Acta Oncol. 52(8), 1593-1601 (2013).

34. Shore ND, Chowdhury S, Villers A et al. Efficacy and safety of enzalutamide versus bicalutamide for patients with metastatic prostate cancer (TERRAIN): a randomised, double-blind, Phase 2 study. Lancet Oncol. 17(2), 153-163 (2016).

35. Penson DF, Armstrong AJ, Concepcion R et al. Enzalutamide versus bicalutamide in castration-resistant prostate cancer: the STRIVE trial. J. Clin. Oncol. 34(18), 2098-2106 (2016).

36. Beer TM, Armstrong AJ, Rathkopf DE et al. Enzalutamide in metastatic prostate cancer before chemotherapy. N. Engl. J. Med. 371(5), 424-433 (2014).

- Primary reporting of the Phase III PREVAIL trial showing the safety and efficacy of enzalutamide among patients with castration- resistant prostate cancer who have not received chemotherapy.

37. Akaza H, Uemura H, Tsukamoto T et al. A multicenter Phase I/II study of enzalutamide in Japanese patients with castration-resistant prostate cancer. Int. J. Clin. Oncol. 21(4), 773-782 (2016).

38. Morris MJ, Rathkopf DE, Novotny W et al. Phase Ib study of enzalutamide in combination with docetaxel in men with metastatic castration-resistant prostate cancer. Clin. Cancer Res. 22(15), 3774-3781 (2016).

39. de Bono JS, Chowdhury S, Feyerabend $S$ et al. Antitumour activity and safety of enzalutamide in patients with metastatic castration-resistant prostate cancer previously treated with abiraterone acetate plus prednisone for $\geq 24$ weeks in Europe. Eur. Urol. 74(1), 37-45 (2018).

40. Attard G, Borre $\mathrm{M}$, Gurney $\mathrm{H}$ et al. Abiraterone alone or in combination with enzalutamide in metastatic castration-resistant prostate cancer with rising prostate-specific antigen during enzalutamide treatment. J. Clin. Oncol. 36(25), 2639-2646 (2018).

41. Sternberg CN, de Bono JS, Chi KN et al. Improved outcomes in elderly patients with metastatic castration-resistant prostate cancer treated with the androgen receptor inhibitor enzalutamide: results from the Phase III AFFIRM trial. Ann. Oncol. 25(2), 429-434 (2014).

42. Graff JN, Baciarello G, Armstrong AJ et al. Efficacy and safety of enzalutamide in patients 75 years or older with chemotherapy-naive metastatic castration-resistant prostate cancer: results from PREVAIL. Ann. Oncol. 27(2), 286-294 (2016).

43. Siemens DR, Klotz L, Heidenreich A et al. Efficacy and safety of enzalutamide vs bicalutamide in younger and older patients with metastatic castration resistant prostate cancer in the TERRAIN trial. J. Urol. 199(1), 147-154 (2018). 
44. Eisenhauer EA, Therasse P, Bogaerts J et al. New response evaluation criteria in solid tumours: revised RECIST guideline (version 1.1). Eur. J. Cancer 45(2), 228-247 (2009).

45. Therasse P, Arbuck SG, Eisenhauer EA et al. New guidelines to evaluate the response to treatment in solid tumors. European Organization for Research and Treatment of Cancer, National Cancer Institute of the United States, National Cancer Institute of Canada. J. Natl Cancer Inst. 92(3), 205-216 (2000).

46. Saad F, de Bono J, Shore N et al. Efficacy outcomes by baseline prostate-specific antigen quartile in the AFFIRM trial. Eur. Urol. 67(2), 223-230 (2015).

47. Loriot Y, Fizazi K, de Bono JS, Forer D, Hirmand M, Scher HI. Enzalutamide in castration-resistant prostate cancer patients with visceral disease in the liver and/or lung: outcomes from the randomized controlled Phase III AFFIRM trial. Cancer 123(2), 253-262 (2017).

48. Rathkopf DE, Beer TM, Loriot Y et al. Radiographic progression-free survival as a clinically meaningful end point in metastatic castration-resistant prostate cancer: the PREVAIL randomized clinical trial. JAMA Oncol. 4(5), 694-701 (2018).

49. Beer TM, Armstrong AJ, Rathkopf $\mathrm{D}$ et al. Enzalutamide in men with chemotherapy-naive metastatic castration-resistant prostate cancer: extended analysis of the Phase III PREVAIL study. Eur. Urol. 71(2), 151-154 (2017).

50. Kim CS, Theeuwes A, Kwon DD et al. The PREVAIL trial of enzalutamide in men with chemotherapy-naive, metastatic castration-resistant prostate cancer: post hoc analysis of Korean patients. Investig. Clin. Urol. 57(3), 174-183 (2016).

51. Kimura G, Ueda T. Post hoc analysis of Japanese patients from the placebo-controlled PREVAIL trial of enzalutamide in patients with chemotherapy-naive, metastatic castration-resistant prostate cancer-updated results. Jpn J. Clin. Oncol. 47(3), 262-264 (2017).

52. Evans CP, Higano CS, Keane $\mathrm{T}$ et al. The PREVAIL study: primary outcomes by site and extent of baseline disease for enzalutamide-treated men with chemotherapy-naive metastatic castration-resistant prostate cancer. Eur. Urol. 70(4), 675-683 (2016)

53. Alumkal JJ, Chowdhury S, Loriot $Y$ et al. Effect of visceral disease site on outcomes in patients with metastatic castration-resistant prostate cancer treated with enzalutamide in the PREVAIL trial. Clin. Genitourin. Cancer 15(5), 610-617.e613 (2017).

54. Bryce AH, Alumkal JJ, Armstrong A et al. Radiographic progression with nonrising PSA in metastatic castration-resistant prostate cancer: post hoc analysis of PREVAIL. Prostate Cancer Prostatic Dis. 20(2), 221-227 (2017).

55. Taplin ME, Armstrong AJ, Lin P et al. Clinical outcomes of chemotherapy naive men with metastatic castration resistant prostate cancer and low baseline prostate specific antigen treated with enzalutamide vs placebo. J. Urol. 198(6), 1324-1332 (2017).

56. Cella D, Traina S, Li T et al. Relationship between patient-reported outcomes and clinical outcomes in metastatic castration-resistant prostate cancer: post hoc analysis of COU-AA-301 and COU-AA-302. Ann. Oncol. 29(2), 392-397 (2018).

57. Fizazi K, Scher HI, Miller K et al. Effect of enzalutamide on time to first skeletal-related event, pain, and quality of life in men with castration-resistant prostate cancer: results from the randomised, Phase III AFFIRM trial. Lancet Oncol. 15(10), 1147-1156 (2014).

58. Cella D, Ivanescu C, Holmstrom S, Bui CN, Spalding J, Fizazi K. Impact of enzalutamide on quality of life in men with metastatic castration-resistant prostate cancer after chemotherapy: additional analyses from the AFFIRM randomized clinical trial. Ann. Oncol. 26(1), 179-185 (2015).

59. Loriot $\mathrm{Y}$, Miller $\mathrm{K}$, Sternberg $\mathrm{CN}$ et al. Effect of enzalutamide on health-related quality of life, pain, and skeletal-related events in asymptomatic and minimally symptomatic, chemotherapy-naive patients with metastatic castration-resistant prostate cancer (PREVAIL): results from a randomised, Phase III trial. Lancet Oncol. 16(5), 509-521 (2015).

60. Heidenreich A, Chowdhury S, Klotz L et al. Impact of enzalutamide compared with bicalutamide on quality of life in men with metastatic castration-resistant prostate cancer: additional analyses from the TERRAIN randomised clinical trial. Eur. Urol. 71(4), 534-542 (2017).

61. Devlin N, Herdman M, Pavesi M et al. Health-related quality of life effects of enzalutamide in patients with metastatic castration-resistant prostate cancer: an in-depth post hoc analysis of EQ-5D data from the PREVAIL trial. Health Qual. Life Outcomes 15(1), doi:10.1186/s12955-017-0704-y (2017).

62. Saad F, Ivanescu C, Phung D et al. Skeletal-related events significantly impact health-related quality of life in metastatic castration-resistant prostate cancer: data from PREVAIL and AFFIRM trials. Prostate Cancer Prostatic Dis. 20(1), 110-116 (2017).

63. Joshua AM, Shore ND, Saad F et al. Safety of enzalutamide in patients with metastatic castration-resistant prostate cancer previously treated with docetaxel: expanded access in North America. Prostate 75(8), 836-844 (2015).

64. Xtandi $40 \mathrm{mg}$ soft capsules. Summary of product characteristics. European Medicines Agency (2013) www.ema.europa.eu/docs/en_GB/document_library/EPAR_-_Product_Information/human/002639/WC500144996.pdf

65. Slovin S, Clark W, Carles J et al. Seizure rates in enzalutamide-treated men with metastatic castration-resistant prostate cancer and risk of seizure: the UPWARD study. JAMA Oncol. 4(5), 702-706 (2018).

66. Halabi S, Lin CY, Kelly WK et al. Updated prognostic model for predicting overall survival in first-line chemotherapy for patients with metastatic castration-resistant prostate cancer. J. Clin. Oncol. 32(7), 671-677 (2014).

67. Petrylak DP, Ankerst DP, Jiang CS et al. Evaluation of prostate-specific antigen declines for surrogacy in patients treated on SWOG 99-16. J. Natl Cancer Inst. 98(8), 516-521 (2006). 
68. Armstrong AJ, Garrett-Mayer E, Ou Yang YC et al. Prostate-specific antigen and pain surrogacy analysis in metastatic hormone-refractory prostate cancer. J. Clin. Oncol. 25(25), 3965-3970 (2007).

69. Xu XS, Ryan CJ, Stuyckens $\mathrm{K}$ et al. Correlation between prostate-specific antigen kinetics and overall survival in abiraterone acetate-treated castration-resistant prostate cancer patients. Clin. Cancer Res. 21(14), 3170-3177 (2015).

70. Fuerea A, Baciarello G, Patrikidou A et al. Early PSA response is an independent prognostic factor in patients with metastatic castration-resistant prostate cancer treated with next-generation androgen pathway inhibitors. Eur. J. Cancer 6144-6151 (2016).

71. Armstrong AJ, Saad F, Phung et al. Clinical outcomes and survival surrogacy studies of prostate-specific antigen declines following enzalutamide in men with metastatic castration-resistant prostate cancer previously treated with docetaxel. Cancer 123(12), 2303-2311 (2017).

72. Armstrong AJ, Lin P, Higano CS et al. Prognostic association of prostate-specific antigen decline with clinical outcomes in men with metastatic castration-resistant prostate cancer treated with enzalutamide in a randomized clinical trial. Eur. Urol. Oncol. doi:https://doi.org/10.1016/j.euo.2018.11.005 (2018) (In press).

73. Armstrong AJ, Lin P, Higano CS et al. Development and validation of a prognostic model for overall survival in chemotherapy-naive men with metastatic castration-resistant prostate cancer. Ann. Oncol. 29(11), 2200-2207 (2018).

74. Efstathiou E, Titus M, Wen $S$ et al. Molecular characterization of enzalutamide-treated bone metastatic castration-resistant prostate cancer. Eur. Urol. 67(1), 53-60 (2015).

75. Nakazawa M, Antonarakis ES, Luo J. Androgen receptor splice variants in the era of enzalutamide and abiraterone. Horm. Cancer 5(5), 265-273 (2014).

76. Azad AA, Volik SV, Wyatt AW et al. Androgen receptor gene aberrations in circulating cell-free DNA: biomarkers of therapeutic resistance in castration-resistant prostate cancer. Clin. Cancer Res. 21(10), 2315-2324 (2015).

77. Joseph JD, Lu N, Qian J et al. A clinically relevant androgen receptor mutation confers resistance to second-generation antiandrogens enzalutamide and ARN-509. Cancer Discov. 3(9), 1020-1029 (2013).

78. Korpal M, Korn JM, Gao X et al. An F876L mutation in androgen receptor confers genetic and phenotypic resistance to MDV3100 (enzalutamide). Cancer Discov. 3(9), 1030-1043 (2013).

79. Tucci M, Zichi C, Buttigliero C, Vignani F, Scagliotti GV, Di Maio M. Enzalutamide-resistant castration-resistant prostate cancer: challenges and solutions. OncoTargets Ther. 11, 7353-7368 (2018).

- A comprehensive review of resistence mechanisms to enzalutamide treatment.

80. Poole A, Gill D, Hahn AW et al. Incidence and characterization of antiandrogen withdrawal syndrome after discontinuation of treatment with enzalutamide in castration-resistant prostate cancer. Clin. Genitourin. Cancer doi:10.1016./j.clgc.2017.08.017 (2018) (Epub ahead of print).

81. Baciarello G, Sternberg CN. Treatment of metastatic castration-resistant prostate cancer (mCRPC) with enzalutamide. Crit. Rev. Oncol. Hematol. 106, 14-24 (2016).

82. Penson DF, Lin DW, Karsh L et al. Treatment registry for outcomes in patients with castration-resistant prostate cancer (TRUMPET): a methodology for real-world evidence and research. Future Oncol. 12(23), 2689-2699 (2016). 
